\title{
GEOMETRIC CHARACTERIZATION OF LYAPUNOV EXPONENTS FOR RIEMANN SURFACE LAMINATIONS
}

\author{
VIÊT-ANH NGUYÊN \\ Dedicated to the memory of Gennadi M. Henkin
}

\begin{abstract}
We characterize geometrically the Lyapunov exponents of a cocycle (of arbitrary rank) with respect to a harmonic current defined on a hyperbolic Riemann surface lamination. Our characterizations are formulated in terms of the expansion rates of the cocycle along geodesic rays.
\end{abstract}

Classification AMS 2010: Primary: 37A30, 57R30; Secondary: 58J35, 58J65, $60 \mathrm{~J} 65$. Keywords: Riemann surface lamination, harmonic current, cocycle, Lyapunov exponents, geodesic ray, expansion rate.

\section{INTRODUCTION}

The present work is motivated by the interplay between geometry, topology and dynamics in the theory of hyperbolic Riemann surface laminations. This class of laminations has been extensively studied by numerous authors from different viewpoints and using various methods. The reader is invited to consult the surveys by Fornæss-Sibony [19] and by Ghys [21] as well as the references therein for a recent account on this subject.

To start with we fix some notation and terminology. We refer the reader to Definition 2.1 below for the notion of hyperbolic Riemann surface laminations. Throughout this work $\mathbb{D}$ denotes the unit disc in $\mathbb{C}$ and $g_{P}$ is the Poincaré metric on $\mathbb{D}$, given by

$$
g_{P}(\zeta):=\frac{2}{\left(1-|\zeta|^{2}\right)^{2}} i d \zeta \wedge d \bar{\zeta}, \quad \zeta \in \mathbb{D}, \quad \text { where } \quad i:=\sqrt{-1}
$$

Let $(X, \mathscr{L})$ be a hyperbolic Riemann surface lamination. We emphasize that $X$ is not necessarily compact. For any point $x \in X$, let $L_{x}$ be the leaf passing through $x$ and consider a universal covering map

$$
\phi_{x}: \mathbb{D} \rightarrow L_{x} \quad \text { such that } \phi_{x}(0)=x .
$$

This map is uniquely defined by $x$ up to a rotation on $\mathbb{D}$. Then, by pushing forward the Poincaré metric $g_{P}$ on $\mathbb{D}$ via $\phi_{x}$, we obtain the so-called Poincaré metric on $L_{x}$ which depends only on the leaf. The latter metric is given by a positive $(1,1)$-form on $L_{x}$ that we also denote by $g_{P}$ for the sake of simplicity. A subset $M \subset X$ is called leafwise saturated if $x \in M$ implies $L_{x} \subset M$.

To the lamination $(X, \mathscr{L})$ we associate several objects of different nature. On the dynamical side, consider the sample-path space $\Omega$ which describes the leafwise Brownian motion. Namely, let $\Omega:=\Omega(X, \mathscr{L})$ be the space consisting of all continuous paths $\omega$ : $\mathbb{R}^{+}:=[0, \infty) \rightarrow X$ with image fully contained in a single leaf. Consider the semi-group 
$\left(\sigma_{t}\right)_{t \in \mathbb{R}^{+}}$of shift-transformations $\sigma_{t}: \Omega \rightarrow \Omega$ defined for all $t, s \in \mathbb{R}^{+}$by

$$
\sigma_{t}(\omega)(s):=\omega(s+t), \quad \omega \in \Omega .
$$

For $x \in X$, let $\Omega_{x}$ be the subspace consisting of all paths $\omega$ in $\Omega$ starting from $x$, i.e., $\omega(0)=x$. We endow $\Omega_{x}$ with a canonical probability measure: the Wiener measure $W_{x}$ with respect to the metric $g_{P}$ on $L_{x}$ (see Subsection 2.3 below).

As objects of topological nature, we deal with (multiplicative) cocycles which have been introduced in a previous work [26]. Prior to their formal definition, we make the following convention. Throughout the article, $\mathbb{K}$ denotes either $\mathbb{R}$ or $\mathbb{C}$. Moreover, given any integer $d \geq 1, \operatorname{GL}(d, \mathbb{K})$ denotes the general linear group of degree $d$ over $\mathbb{K}$ and $\mathbb{P}^{d}(\mathbb{K})$ denotes the $\mathbb{K}$-projective space of dimension $d$.

Definition 1.1. A $\mathbb{K}$-valued cocycle (of rank $d$ ) is a map $\mathcal{A}: \Omega \times \mathbb{R}^{+} \rightarrow \operatorname{GL}(d, \mathbb{K})$ such that (1) (identity law) $\mathcal{A}(\omega, 0)=$ id for all $\omega \in \Omega$;

(2) (homotopy law) if $\omega_{1}, \omega_{2} \in \Omega_{x}$ and $t_{1}, t_{2} \in \mathbb{R}^{+}$such that $\omega_{1}\left(t_{1}\right)=\omega_{2}\left(t_{2}\right)$ and $\left.\omega_{1}\right|_{\left[0, t_{1}\right]}$ is homotopic to $\left.\omega_{2}\right|_{\left[0, t_{2}\right]}$ (that is, the path $\left.\omega_{1}\right|_{\left[0, t_{1}\right]}$ can be deformed continuously on $L_{x}$ to the path $\left.\omega_{2}\right|_{\left[0, t_{2}\right]}$, the two endpoints of $\left.\omega_{1}\right|_{\left[0, t_{1}\right]}$ being kept fixed during the deformation), then

$$
\mathcal{A}\left(\omega_{1}, t_{1}\right)=\mathcal{A}\left(\omega_{2}, t_{2}\right) ;
$$

(3) (multiplicative law) $\mathcal{A}(\omega, s+t)=\mathcal{A}\left(\sigma_{t}(\omega), s\right) \mathcal{A}(\omega, t)$ for all $s, t \in \mathbb{R}^{+}$and $\omega \in \Omega$;

(4) (measurable law) the local expression of $\mathcal{A}$ on each laminated chart is Borel measurable (see Subsection 2.1 below for the definition of local expressions).

It is worthy noting that the cocycles of rank 1 have been investigated by several authors (see, for example, Candel [7], Deroin [12], etc). The holonomy cocycle (or equivalently the normal derivative cocycle) of the regular part of a $n$-dimensional (possibly singular) holomorphic foliation by hyperbolic Riemann surfaces provides a typical example of $\mathbb{C}$-valued cocycles of rank $n-1$. Another source of cocycles are those associated with foliations which are obtained from suspensions. These cocycles capture the topological aspect of the considered foliations. Moreover, we can produce new cocycles from the old ones by performing some basic operations such as the wedge product and the tensor product (see [26, Section 3.1]). In this article, we are mainly concerned with cocycles which behave in a tempered manner relative to the metric $g_{P}$. More concretely, we will introduce in Definition 2.8 below two large families of cocycles: the moderate cocycles and the Hölder ones.

The objects of geometric nature considered here are harmonic currents given by Garnett [20] which are generalizations of the foliations cycles previously introduced by Sullivan [30]. When $X$ is compact, the existence of non-zero harmonic currents $T$ has been established by Garnett [20]. The case when $X$ is non compact has been investigated by Berndtsson-Sibony and Fornæss-Sibony [3, 19]. To a non-zero harmonic current $T$ we associate a Borel positive measure

$$
\mu=\mu_{T}:=T \wedge g_{P}
$$

which is also a harmonic measure in good cases (for example, when $X$ is compact, see Proposition 2.10 below). The notion of harmonic measures and harmonic currents will be recalled in Definition 2.4 and 2.5. The following terminology will be repeatedly used in this article. Given a positive finite measure space $(S, \mathscr{S}, \nu)$, a set $A \in \mathscr{S}$ is called of full 
$\nu$-measure if $\nu(S \backslash A)=0$. In what follows, we say that a set $A \subset X$ is of full T-measure for a harmonic current $T$ if it is of full $\mu$-measure, where $\mu$ is given in (2).

In this setting, using our recent work [26] we obtain the following Oseledec multiplicative ergodic theorem which relates different objects of different natures.

Theorem 1.2. Let $(X, \mathscr{L})$ be a $\mathscr{C}^{2}$-smooth hyperbolic Riemann surface lamination and $T$ a harmonic current. Let $\mu$ be the measure associated to $T$ by (2). Assume, moreover, that $T$ is extremal in the cone of all harmonic currents and that $\mu$ is a (finite) harmonic measure. Consider a moderate cocycle $\mathcal{A}: \Omega \times \mathbb{R}^{+} \rightarrow \mathrm{GL}(d, \mathbb{K})$. Then there exist a leafwise saturated Borel set $Y \subset X$ of full $\mu$-measure and a number $m \in \mathbb{N}$ together with $m$ integers $d_{1}, \ldots, d_{m} \in \mathbb{N}$ such that the following properties hold:

(i) For each $x \in Y$ there exists a decomposition of $\mathbb{K}^{d}$ as a direct sum of $\mathbb{K}$-linear subspaces

$$
\mathbb{K}^{d}=\oplus_{i=1}^{m} H_{i}(x),
$$

such that $\operatorname{dim} H_{i}(x)=d_{i}$ and $\mathcal{A}(\omega, t) H_{i}(x)=H_{i}(\omega(t))$ for all $\omega \in \Omega_{x}$ and $t \in \mathbb{R}^{+}$. Moreover, $x \mapsto H_{i}(x)$ is a measurable map from $Y$ into the Grassmannian of $\mathbb{K}^{d}$. For each $1 \leq i \leq m$ and each $x \in Y$, let $V_{i}(x):=\oplus_{j=i}^{m} H_{j}(x)$. Set $V_{m+1}(x) \equiv\{0\}$.

(ii) There are real numbers

$$
\chi_{m}<\chi_{m-1}<\cdots<\chi_{2}<\chi_{1},
$$

and for each $x \in Y$, there is a set $F_{x} \subset \Omega_{x}$ of full $W_{x}$-measure such that for every $1 \leq i \leq m$ and every $v \in V_{i}(x) \backslash V_{i+1}(x)$ and every $\omega \in F_{x}$,

$$
\lim _{t \rightarrow \infty, t \in \mathbb{R}^{+}} \frac{1}{t} \log \frac{\|\mathcal{A}(\omega, t) v\|}{\|v\|}=\chi_{i} .
$$

Moreover,

$$
\lim _{t \rightarrow \infty, t \in \mathbb{R}^{+}} \frac{1}{t} \log \|\mathcal{A}(\omega, t)\|=\chi_{1}
$$

for each $x \in Y$ and for every $\omega \in F_{x}$.

Here $\|\cdot\|$ denotes the standard Euclidean norm of $\mathbb{K}^{d}$.

The above result is the counterpart, in the context of hyperbolic Riemann surface laminations, of the classical Oseledec multiplicative ergodic theorem for maps (see [23, 29]).

Assertion (i) above says that the Oseledec decomposition exists for all points $x$ in a leafwise saturated Borel set of full $\mu$-measure and that this decomposition is holonomy invariant. It is worthy noting that the Oseledec decomposition in (i) depends only on $x \in Y$, in particular, it does not depend on paths $\omega \in \Omega_{x}$.

The decreasing sequence of subspaces of $\mathbb{K}^{d}$ given by assertion (i):

$$
\{0\} \equiv V_{m+1}(x) \subset V_{m}(x) \subset \cdots \subset V_{1}(x)=\mathbb{K}^{d}
$$

is called the Lyapunov filtration associated to $\mathcal{A}$ at a given point $x \in Y$.

The numbers $\chi_{m}<\chi_{m-1}<\cdots<\chi_{2}<\chi_{1}$ given by assertion (ii) above are called the Lyapunov exponents of the cocycle $\mathcal{A}$ with respect to the current $T$. Moreover, we infer from formulas (3) and (4) above that these characteristic numbers measure heuristically the expansion rate of $\mathcal{A}$ along different vector-directions $v$ and along leafwise Brownian 
trajectories. In other words, the stochastic formulas (3)-(4) only express the dynamical character of the Lyapunov exponents.

The main purpose of this work is to find a geometric interpretation of these characteristic quantities. Our approach consists in replacing the Brownian trajectories by the more appealing objects, namely, the unit-speed geodesic rays. These paths are parameterized by their length (with respect to the leafwise Poincaré metric). Therefore, we characterize the Lyapunov exponents in terms of the expansion rates of $\mathcal{A}$ along the geodesic rays.

For this purpose we need to introduce some more notation and terminology. Denote by $r \mathbb{D}$ the disc in $\mathbb{C}$ of center 0 and of radius $r$ with $0<r<1$. In the Poincaré disc $\left(\mathbb{D}, \omega_{P}\right), r \mathbb{D}$ is also the disc of center 0 and of radius

$$
R:=\log \frac{1+r}{1-r} .
$$

So, we will also denote by $\mathbb{D}_{R}$ this disc and by $\partial \mathbb{D}_{R}$ its boundary. Conversely, for each $R>0$ we denote by $r_{R}$ the unique number $0<r<1$ satisfying the above equation, that is, $r_{R} \mathbb{D}=\mathbb{D}_{R}$.

Recall from (1) that $\left(\phi_{x}\right)_{x \in X}$ is a given family of universal covering maps $\phi_{x}: \mathbb{D} \rightarrow L_{x}$ with $\phi_{x}(0)=x$. For every $x \in X$, the set of all unit-speed geodesic rays $\omega:[0, \infty) \rightarrow L_{x}$ starting at $x$ (that is, $\omega(0)=x$ ), can be described by the family $\left(\gamma_{x, \theta}\right)_{\theta \in[0,1)}$, where

$$
\gamma_{x, \theta}(R):=\phi_{x}\left(e^{2 \pi i \theta} r_{R}\right), \quad R \in \mathbb{R}^{+} .
$$

The path $\gamma_{x, \theta}$ is called the unit-speed geodesic ray at $x$ with the leaf-direction $\theta$. Unless otherwise specified, the space of leaf-directions $[0,1)$ is endowed with the Lebesgue measure. The space of leaf-directions is visibly identified, via the map $[0,1) \ni \theta \mapsto e^{2 \pi i \theta}$, with the unit circle $\partial \mathbb{D}$ endowed with the normalized rotation measure.

In order to state our main results, the following notions of expansion rates for cocycles are needed.

Definition 1.3. Let $\mathcal{A}$ be a $\mathbb{K}$-valued cocycle and $R>0$ a time.

The expansion rate of $\mathcal{A}$ at a point $x \in X$ in the leaf-direction $\theta$ at time $R$ along the vector $v \in \mathbb{K}^{d} \backslash\{0\}$ is the number

$$
\mathscr{E}(x, \theta, v, R):=\frac{1}{R} \log \frac{\left\|\mathcal{A}\left(\gamma_{x, \theta}, R\right) v\right\|}{\|v\|} .
$$

The expansion rate of $\mathcal{A}$ at a point $x \in X$ in the leaf-direction $\theta$ at time $R$ is

$$
\begin{aligned}
\mathscr{E}(x, \theta, R):=\sup _{v \in \mathbb{K}^{d} \backslash\{0\}} \mathscr{E}(x, \theta, v, R) & =\sup _{v \in \mathbb{K}^{d} \backslash\{0\}} \frac{1}{R} \log \frac{\left\|\mathcal{A}\left(\gamma_{x, \theta}, R\right) v\right\|}{\|v\|} \\
& =\frac{1}{R} \log \left\|\mathcal{A}\left(\gamma_{x, \theta}, R\right)\right\| .
\end{aligned}
$$

Given a $\mathbb{K}$-vector subspace $\{0\} \neq H \subset \mathbb{K}^{d}$, the expansion rate of $\mathcal{A}$ at a point $x \in X$ at time $R$ along the vector space $H$ is the interval $\mathscr{E}(x, H, R):=[a, b]$, where

$$
a:=\inf _{v \in H \backslash\{0\}} \int_{0}^{1}\left(\frac{1}{R} \log \frac{\left\|\mathcal{A}\left(\gamma_{x, \theta}, R\right) v\right\|}{\|v\|}\right) d \theta \text { and } b:=\sup _{v \in H \backslash\{0\}} \int_{0}^{1}\left(\frac{1}{R} \log \frac{\left\|\mathcal{A}\left(\gamma_{x, \theta}, R\right) v\right\|}{\|v\|}\right) d \theta \text {. }
$$

Notice that $\mathscr{E}(x, \theta, v, R)$ (resp. $\mathscr{E}(x, \theta, R)$ ) expresses geometrically the expansion rate (resp. the maximal expansion rate) of the cocycle when one travels along the unit-speed 
geodesic ray $\gamma_{x, \theta}$ up to time $R$. On the other hand, $\mathscr{E}(x, H, R)$ represents the smallest closed interval which contains all numbers

$$
\int_{0}^{1}\left(\frac{1}{R} \log \frac{\left\|\mathcal{A}\left(\gamma_{x, \theta}, R\right) v\right\|}{\|v\|}\right) d \theta
$$

where $v$ ranges over $H \backslash\{0\}$. Note that the above integral is the average of the expansion rate of the cocycle when one travels along the unit-speed geodesic rays along the vectordirection $v \in H$ from $x$ to the Poincaré circle with radius $R$ and center $x$ spanned on $L_{x}$.

We say that a sequence of intervals $[a(R), b(R)] \subset \mathbb{R}$ indexed by $R \in \mathbb{R}^{+}$converges to a number $\chi \in \mathbb{R}$ and write $\lim _{R \rightarrow \infty}[a(R), b(R)]=\chi$, if $\lim _{R \rightarrow \infty} a(R)=\lim _{R \rightarrow \infty} b(R)=\chi$.

Now we are able to state the main result.

Theorem 1.4. (Main Theorem). Let $(X, \mathscr{L})$ be a $\mathscr{C}^{2}$-smooth hyperbolic Riemann surface lamination and $T$ a harmonic current. Let $\mu$ be the measure associated to $T$ by (2). Assume, moreover, that $T$ is extremal and that $\mu$ is a (finite) harmonic measure. Consider a moderate cocycle $\mathcal{A}: \Omega \times \mathbb{R}^{+} \rightarrow \mathrm{GL}(d, \mathbb{K})$. Then there is a leafwise saturated Borel set $Y$ of full T-measure which satisfies the conclusion of Theorem 1.2 and the following additional properties:

(i) Assume that $\mathcal{A}$ is Hölder of order $\alpha<2$. Then, for each $1 \leq i \leq m$ and for each $x \in Y$, there is a set $G_{x} \subset[0,1)$ of full Lebesgue measure such that for each $v \in$ $V_{i}(x) \backslash V_{i+1}(x)$,

$$
\lim _{R \rightarrow \infty} \mathscr{E}(x, \theta, v, R)=\chi_{i}, \quad \theta \in G_{x}
$$

Moreover, the maximal Lyapunov exponent $\chi_{1}$ satisfies

$$
\lim _{R \rightarrow \infty} \mathscr{E}(x, \theta, R)=\chi_{1}, \quad \theta \in G_{x} .
$$

(ii) Assume that $\mathcal{A}$ is strongly moderate. Then, for each $1 \leq i \leq m$ and each $x \in Y$,

$$
\lim _{R \rightarrow \infty} \mathscr{E}\left(x, H_{i}(x), R\right)=\chi_{i} .
$$

Here $\mathbb{K}^{d}=\oplus_{i=1}^{m} H_{i}(x), x \in Y$, is the Oseledec decomposition given by Theorem 1.2 and $\chi_{m}<\chi_{m-1}<\cdots<\chi_{2}<\chi_{1}$ are the corresponding Lyapunov exponents.

Theorem 1.4 gives a geometric meaning to the stochastic formulas (3)-(4).

Applying Theorem 1.4 to the case where $X$ is compact and the cocycle $\mathcal{A}$ is $\mathscr{C}^{2}$ differentiable (see Proposition 2.10 below), we obtain the following

Corollary 1.5. Let $(X, \mathscr{L})$ be a $\mathscr{C}^{2}$-smooth hyperbolic Riemann surface lamination and $T$ a harmonic current. Assume, moreover, that $X$ is compact and $T$ is extremal. Consider a $\mathscr{C}^{2}$-differentiable cocycle $\mathcal{A}: \Omega \times \mathbb{R}^{+} \rightarrow \mathrm{GL}(d, \mathbb{K})$. Then there is a leafwise saturated Borel set $Y$ of full $T$-measure which satisfies the conclusions of Theorem 1.2 as well as those of Theorem 1.4.

Let $(M, \mathscr{L}, E)$ be a transversally $\mathscr{C}^{2}$-smooth (resp. transversally holomorphic) singular foliation by Riemann surfaces with the set of singularities $E$ in a Riemannian manifold (resp. Hermitian complex manifold) $M$. Consider a leafwise saturated, compact set $X \subset M \backslash E$ whose leaves are all hyperbolic. So the restriction of the foliation $(M \backslash E, \mathscr{L})$ to $X$ gives an inherited compact $\mathscr{C}^{2}$-smooth hyperbolic Riemann lamination $(X, \mathscr{L})$. 
Moreover, the holonomy cocycle of $(M \backslash E, \mathscr{L})$ induces, by restriction, an inherited $\mathscr{C}^{2}$ differentiable cocycle on $(X, \mathscr{L})$ (see Example 2.11 below). Hence, Corollary 1.5 applies to the latter cocycle. In particular, when $(M, \mathscr{L}, E)$ is a transversally holomorphic singular foliation on a compact Hermitian complex manifold $M$ of dimension $n$, the corollary applies to the induced holonomy cocycle of rank $n-1$ associated with every minimal set $X$ whose leaves are all hyperbolic. Here a minimal set is a leafwise saturated closed subset of $M$ which contains no proper subset with this property.

We outline the strategy of the proofs of Theorem 1.2 and Theorem 1.4. In the previous work [26, Theorem 3.7 and 3.11], we have developed a general approach to obtain Oseledec multiplicative ergodic theorems for general laminations. For the proof of Theorem 1.2 we adapt this approach to the present context of hyperbolic Riemann surface laminations.

The proof of Theorem 1.4 constitutes the core of this article. The proof of its first part relies on the theory of Brownian trajectories on hyperbolic spaces. More concretely, some quantitative results on the boundary behavior of Brownian trajectories by Lyons [24] and Cranston [11] and on the shadow of Brownian trajectories by geodesic rays (see, for example, Ancona [2]) are our main ingredients. This, combined with the Hölder regularity of the cocycle, allows us to replace a Brownian trajectory by a unit-speed geodesic ray with uniformly distributed leaf-direction. Hence, Part (i) of Theorem 1.4 will follow from Theorem 1.2 .

To establish Part (ii) of Theorem 1.4 we need two steps. In the first step we adapt to our context the so-called Ledrappier type characterization of Lyapunov spectrum which was introduced in the previous work [26]. This, combined with the ratio ergodic theorem due to Akcoglu-Sucheston [1], allows us to show that a similar version of formula (9) holds when the expansion rates in terms of geodesic rays are replaced by some heat diffusions associated with the cocycle.

The second step shows that the above heat diffusions can be approximated by the expansion rates. To do this we establish a new geometric estimate on the heat diffusions (see Lemma[5.3 below). In fact, this delicate estimate relies on the proof of the geometric Birkhoff ergodic theorem developed in a previous joint-work with Dinh and Sibony [14]. Combining the two steps, Part (ii) of Theorem 1.4 follows.

The article is organized as follows. Section 2 sets up the background of this work. Section 3 is devoted to the proof of Theorem 1.2 and Part (i) of Theorem 1.4. The first step in the proof of Part (ii) of Theorem 1.4 is developed in Section 4. The second step is given in Section 5, When the lamination $(X, \mathscr{L})$ and the cocycle $\mathcal{A}$ arise from some particular suspensions, in parallel to our approach there is another classical way to define Lyapunov exponents using the geodesic flows (see, for example, [4]). The last section is devoted to the proof that, in this context, both approaches give the same Oseledec decomposition and the same Lyapunov spectrum. Nevertheless, our method gives more geometric properties than the other one. Moreover, this context corresponds to a very special case of our result when the lamination consists only of a single leaf. The section is concluded with some open questions and remarks.

Acknowledgement. The author would like to thank Alano Ancona for interesting discussions. He also thanks the referee for carefully reading the paper and for suggestions leading to the improvement of the exposition. This work was partially prepared during 
the author's visit at the Max-Planck Institute for Mathematics in Bonn. He would like to express his gratitude to this organization for hospitality and for financial support.

\section{PREPARATORY RESUlts}

2.1. Lamination, hyperbolicity and cocycle. Let $X$ be a locally compact space. A Riemann surface lamination $(X, \mathscr{L})$ is the data of a (lamination) atlas $\mathscr{L}$ of $X$ with (laminated) charts

$$
\Phi_{p}: \mathbb{U}_{p} \rightarrow \mathbb{B}_{p} \times \mathbb{T}_{p}
$$

Here, $\mathbb{T}_{p}$ is a locally compact metric space, $\mathbb{B}_{p}$ is a domain in $\mathbb{C}, \mathbb{U}_{p}$ is an open set in $X$, and $\Phi_{p}$ is a homeomorphism, and all the changes of coordinates $\Phi_{p} \circ \Phi_{q}^{-1}$ are of the form

$$
x=(y, t) \mapsto x^{\prime}=\left(y^{\prime}, t^{\prime}\right), \quad y^{\prime}=\Psi(y, t), \quad t^{\prime}=\Lambda(t),
$$

where $\Psi, \Lambda$ are continuous functions and $\Psi$ is holomorphic in $y$. Moreover, we say that $(X, \mathscr{L})$ is $\mathscr{C}^{k}$-smooth for some $k \in \mathbb{N} \cup\{\infty\}$ if $\Psi$ is $\mathscr{C}^{k}$-smooth with respect to $y$, and its partial derivatives of any total order $\leq k$ with respect to $y$ and $\bar{y}$ are jointly continuous with respect to $(y, t)$.

The open set $\mathbb{U}_{p}$ is called a flow box and the Riemann surface $\Phi_{p}^{-1}\{t=c\}$ in $\mathbb{U}_{p}$ with $c \in \mathbb{T}_{p}$ is a plaque. The property of the above coordinate changes insures that the plaques in different flow boxes are compatible in the intersection of the boxes. Two plaques are adjacent if they have non-empty intersection.

A leaf $L$ is a minimal connected subset of $X$ such that if $L$ intersects a plaque, it contains that plaque. So a leaf $L$ is a Riemann surface immersed in $X$ which is a union of plaques.

Definition 2.1. A leaf $L$ of a lamination $(X, \mathscr{L})$ is said to be hyperbolic if it is a hyperbolic Riemann surface, i.e., it is uniformized by $\mathbb{D}$. The lamination is said to be hyperbolic if its leaves are all hyperbolic.

Standing Hypothesis. From now on, we always assume that $(X, \mathscr{L})$ is a $\mathscr{C}^{2}$-smooth Riemann surface lamination.

We denote by $\mathscr{C}(X, \mathscr{L})$ the space of all functions $f$ defined and compactly supported on $X$ which are leafwise $\mathscr{C}^{2}$-smooth and transversally continuous, that is, for each laminated chart $\Phi_{p}: \mathbb{U}_{p} \rightarrow \mathbb{B}_{p} \times \mathbb{T}_{p}$ and all $m, n \in \mathbb{N}$ with $m+n \leq 2$, the derivatives $\frac{\partial^{m+n}\left(f \circ \Phi_{p}^{-1}\right)}{\partial y^{m} \partial \bar{y}^{n}}$ exist and are jointly continuous in $(y, t)$.

When a lamination $(X, \mathscr{L})$ satisfies that $X$ is a manifold and that the leaves of $\mathscr{L}$ are Riemann surfaces immersed in $X$, we say that $(X, \mathscr{L})$ is a foliation. Moreover, $(X, \mathscr{L})$ is called a transversally $\mathscr{C}^{k}$-smooth foliation (resp. transversally holomorphic foliation when $X$ is a complex manifold) if there is an atlas $\mathscr{L}$ of $X$ with charts

$$
\Phi_{i}: \mathbb{U}_{i} \rightarrow \mathbb{B}_{i} \times \mathbb{T}_{i}
$$

with $\mathbb{T}_{i}$ an open set of some $\mathbb{R}^{d}$ (resp. an open set of some $\mathbb{C}^{d}$ ) such that each above map $\Psi$ is a diffeomorphism of class $\mathscr{C}^{k}$ (resp. a biholomorphic map).

We say that $(M, \mathscr{L}, E)$ is a singular foliation if $M$ is a manifold and $E \subset M$ is a closed subset such that $\overline{M \backslash E}=M$ and $(M \backslash E, \mathscr{L})$ is a foliation. $E$ is said to be the set of singularities. 
Let $\mathcal{A}: \Omega(X, \mathscr{L}) \times \mathbb{R}^{+} \rightarrow \mathrm{GL}(d, \mathbb{K})$ be a map that satisfies the identity, homotopy and multiplicative laws in Definition 1.1. In any chart $\Phi_{p}: \mathbb{U}_{p} \rightarrow \mathbb{B}_{p} \times \mathbb{T}_{p}$ with $\mathbb{B}_{p}$ simply connected, consider the map $A_{p}: \mathbb{B}_{p} \times \mathbb{B}_{p} \times \mathbb{T}_{p} \rightarrow \mathrm{GL}(d, \mathbb{K})$ defined by

$$
A_{p}(y, z, t):=\mathcal{A}(\omega, 1),
$$

where $\omega$ is any leafwise path such that $\omega(0)=\Phi_{p}^{-1}(y, t), \omega(1)=\Phi_{p}^{-1}(z, t)$ and $\omega[0,1]$ is contained in the simply connected plaque $\Phi_{p}^{-1}(\cdot, t)$. Now we are able to explain the last law in Definition 1.1 and single out some new classes of cocycles.

Definition 2.2. $A_{p}$ is called the local expression of $\mathcal{A}$ on the chart $\Phi_{p}$.

$\mathcal{A}$ is called a cocycle if its local expression on each chart is Borel measurable.

Now let $(X, \mathscr{L})$ be a $\mathscr{C}^{k}$-smooth hyperbolic Riemann surface lamination for some $k \in$ $\mathbb{N} \cup\{\infty\}$.

- $\mathcal{A}$ is called a leafwise $\mathscr{C}^{k}$-differentiable cocycle if, for each chart $\Phi_{p}$, the local expression $A_{p}$ is $\mathscr{C}^{k}$-differentiable with respect to $(y, z)$.

- $\mathcal{A}$ is called a $\mathscr{C}^{k}$-differentiable cocycle if, for each chart $\Phi_{p}$, the local expression $A_{p}$ is $\mathscr{C}^{k}$-differentiable with respect to $(y, z)$ and its partial derivatives of any total order $\leq k$ with respect to $(y, z)$ are jointly continuous in $(y, z, t)$.

Example 2.3. A fundamental example of $\mathscr{C}^{k}$-differentiable $\mathbb{R}$-valued (resp. $\mathbb{C}$-valued) cocycles is the holonomy cocycle of a transversally $\mathscr{C}^{k}$-smooth (resp. transversally holomorphic) foliation $(X, \mathscr{L})$ by hyperbolic Riemann surfaces in a Riemannian manifold (resp. Hermitian complex manifold) $X$. See [26, Proposition 3.3] for more details.

A more sophisticated situation will be discussed in Example 2.11 below.

2.2. Heat diffusions and harmonic currents versus harmonic measures. Let $(X, \mathscr{L})$ be a hyperbolic Riemann surface lamination. The leafwise Poincaré metric $g_{P}$ induces the corresponding Laplacian $\Delta$ on leaves (see [14]). For every point $x \in X$ consider the heat equation on $L_{x}$

$$
\frac{\partial p(x, y, t)}{\partial t}=\Delta_{y} p(x, y, t), \quad \lim _{t \rightarrow 0+} p(x, y, t)=\delta_{x}(y), \quad y \in L_{x}, t \in \mathbb{R}_{+} .
$$

Here $\delta_{x}$ denotes the Dirac mass at $x, \Delta_{y}$ denotes the Laplacian $\Delta$ with respect to the variable $y$, and the limit is taken in the sense of distribution, that is,

$$
\lim _{t \rightarrow 0+} \int_{L_{x}} p(x, y, t) f(y) g_{P}(y)=f(x)
$$

for every smooth function $f$ compactly supported in $L_{x}$.

The smallest positive solution of the above equation, denoted by $p(x, y, t)$, is called the heat kernel. Such a solution exists because $\left(L_{x}, g_{P}\right)$ is complete and of bounded geometry (see, for example, [8, 10]). The heat kernel $p(x, y, t)$ gives rise to a one parameter family $\left\{D_{t}: t \geq 0\right\}$ of diffusion operators defined on bounded measurable functions on $X$ by

$$
D_{t} f(x):=\int_{L_{x}} p(x, y, t) f(y) g_{P}(y), \quad x \in X
$$

We record here the semi-group property of this family:

$$
D_{0}=\mathrm{id} \quad \text { and } \quad D_{t} \mathbf{1}=\mathbf{1} \text { and } D_{t+s}=D_{t} \circ D_{s} \quad \text { for } t, s \geq 0,
$$

where 1 denotes the function which is identically equal to 1 . 
Using the map $\phi_{x}: \mathbb{D} \rightarrow L_{x}$ given in (1), the following identity relates the diffusion operators in $L_{x}$ and those in the Poincaré disc $\left(\mathbb{D}, g_{P}\right)$ : For $x \in X$ and for every bounded measurable function $f$ defined on $L_{x}$,

$$
D_{t}\left(f \circ \phi_{x}\right)=\left(D_{t} f\right) \circ \phi_{x}, \quad \text { on } L_{x} \text { for all } t \in \mathbb{R}^{+} .
$$

See [26, Proposition 2.7] for a proof.

Now we arrive at two notions of harmonic measures.

Definition 2.4. A positive locally finite Borel measure $\mu$ on $X$ is said to be quasi-harmonic if

$$
\int_{X} \Delta u d \mu=0
$$

for all functions $u \in \mathscr{C}(X, \mathscr{L})$.

A quasi-harmonic measure $\mu$ is said to be harmonic if $\mu$ is finite and $\mu$ is $D_{t}$-invariant for all $t \in \mathbb{R}^{+}$, i.e,

$$
\int_{X} D_{t} f d \mu=\int_{X} f d \mu, \quad f \in \mathscr{C}(X, \mathscr{L}), t \in \mathbb{R}^{+} .
$$

Let $\mathscr{C}^{1}(X, \mathscr{L})$ denote the space of all forms $h$ of bidegree $(1,1)$ defined on leaves of the lamination and compactly supported on $X$ such that $h$ is leafwise continuous and transversally continuous, that is, for each laminated chart $\Phi_{p}: \mathbb{U}_{p} \rightarrow \mathbb{B}_{p} \times \mathbb{T}_{p}$, the form $h \circ \Phi_{p}^{-1}$ is jointly continuous in $(y, t)$. For each chart $\Phi_{p}: \mathbb{U}_{p} \rightarrow \mathbb{B}_{p} \times \mathbb{T}_{p}$, the complex structure on $\mathbb{B}_{p}$ induces a complex structure on the leaves of $X$. Therefore, the operator $\partial$ and $\bar{\partial}$ can be defined so that they act leafwise on forms as in the case of manifolds. So we get easily that $\partial \bar{\partial}: \mathscr{C}(X, \mathscr{L}) \rightarrow \mathscr{C}^{1}(X, \mathscr{L})$. A form $h \in \mathscr{C}^{1}(X, \mathscr{L})$ is said to be positive if its restriction to every plaque is a positive $(1,1)$-form in the usual sense of Lelong.

Definition 2.5. A harmonic current $T$ on the lamination is a linear continuous form on $\mathscr{C}^{1}(X, \mathscr{L})$ which verifies $\partial \bar{\partial} T=0$ in the weak sense (namely $T(\partial \bar{\partial} f)=0$ for all $f \in$ $\mathscr{C}(X, \mathscr{L})$ ), and which is positive (namely, $T(h) \geq 0$ for all positive forms $h \in \mathscr{C}^{1}(X, \mathscr{L})$ ).

For the existence of nonzero harmonic currents, see the discussion preceding Theorem 1.2 .

Recall that a positive finite measure $\mu$ on the $\sigma$-algebra of Borel sets in $X$ is said to be ergodic if for every leafwise saturated measurable set $Z \subset X, \mu(Z)$ is equal to either $\mu(X)$ or 0 . A harmonic current $T$ is said to be extremal if it is an extremal point in the convex cone of all harmonic currents. The following result relates the notions of harmonic measures and harmonic currents.

Theorem 2.6. Let $(X, \mathscr{L})$ be a hyperbolic Riemann surface lamination.

(i) If $X$ is compact, then each quasi-harmonic measure is harmonic.

(ii) The map $T \mapsto \mu=T \wedge g_{P}$ which is defined on the convex cone of all harmonic currents is one-to-one and its image is contained in the convex cone of all quasi-harmonic measures $\mu$. If, moreover, $X$ is compact, then this map is an one-to-one correspondence between the convex cone of all harmonic currents $T$ and the convex cone of all harmonic measures $\mu$.

(iii) If $T$ is an extremal harmonic current and $\mu:=T \wedge g_{P}$ is finite, then $\mu$ is ergodic.

Proof. Assertion (i) follows from the theory developed in [20] (see also [8, Proposition

2.4.2] and [14, Theorem 5.7] for more explicit proofs). 
The first part of assertion (ii) follows from Definition 2.4 and 2.5, When $X$ is compact, we know, by [14, Proposition 5.1], that the map $T \mapsto \mu=T \wedge g_{P}$ is an one-to-one correspondence between the convex cone of harmonic currents $T$ and the convex cone of quasi-harmonic measures $\mu$. This, combined with assertion (i), completes the second part of assertion (ii).

To prove assertion (iii), suppose in order to get a contradiction that $\mu$ is not ergodic. So there is a leafwise saturated Borel set $A \subset X$ such that $0<\mu(A)<\mu(X)$. Let $\mu_{1}:=\left.2 \mu\right|_{A}$ and $\mu_{2}:=\left.2 \mu\right|_{X \backslash A}$. So $\mu=\frac{\mu_{1}+\mu_{2}}{2}$, and $\mu_{1}, \mu_{2}$ are not co-linear. Using the local description of $T$ on each flow box (see [14, Proposition 2.3 ]), we can show that both $\mu_{1}$ and $\mu_{2}$ are quasi-harmonic measures. By the first part of assertion (ii), let $T_{1}, T_{2}$ be harmonic currents such that $\mu_{1}:=T_{1} \wedge g_{P}$ and $\mu_{2}:=T_{2} \wedge g_{P}$. This, combined with $\mu=\frac{\mu_{1}+\mu_{2}}{2}$, implies that $T=\frac{T_{1}+T_{2}}{2}$ and $T_{1}, T_{2}$ are not co-linear. This contradicts the extremality of $T$.

2.3. Wiener measures. In this subsection we follow the expositions given in Section $2.2,2.4$ and 2.5 in our previous work [26], which are, in turn, inspired by Garnett's theory of leafwise Brownian motion in [20] (see also [7, 8]).

We first recall the construction of the Wiener measure $W_{0}$ on the Poincaré disc $\left(\mathbb{D}, g_{P}\right)$. Let $\Omega_{0}$ be the space consisting of all continuous paths $\omega:[0, \infty) \rightarrow \mathbb{D}$ with $\omega(0)=0$. A cylinder set (in $\Omega_{0}$ ) is a set of the form

$$
C=C\left(\left\{t_{i}, B_{i}\right\}: 1 \leq i \leq m\right):=\left\{\omega \in \Omega_{0}: \omega\left(t_{i}\right) \in B_{i}, \quad 1 \leq i \leq m\right\} .
$$

where $m$ is a positive integer and the $B_{i}$ 's are Borel subsets of $\mathbb{D}$, and $0<t_{1}<t_{2}<\cdots<$ $t_{m}$ is a set of increasing times. In other words, $C$ consists of all paths $\omega \in \Omega_{0}$ which can be found within $B_{i}$ at time $t_{i}$. Let $\mathscr{A}_{0}$ be the $\sigma$-algebra on $\Omega_{0}$ generated by all cylinder sets. For each cylinder set $C:=C\left(\left\{t_{i}, B_{i}\right\}: 1 \leq i \leq m\right)$ as above, define

$$
W_{x}(C):=\left(D_{t_{1}}\left(\chi_{B_{1}} D_{t_{2}-t_{1}}\left(\chi_{B_{2}} \cdots \chi_{B_{m-1}} D_{t_{m}-t_{m-1}}\left(\chi_{B_{m}}\right) \cdots\right)\right)\right)(x),
$$

where, $\chi_{B_{i}}$ is the characteristic function of $B_{i}$ and $D_{t}$ is the diffusion operator given by (10) where $p(x, y, t)$ therein is replaced by the heat kernel $\mathbf{p}(\xi, \zeta, t)$ of the Poincaré disc. It is well-known that $W_{0}$ can be extended to a unique probability measure on $\left(\Omega_{0}, \mathscr{A}_{0}\right)$. This is the canonical Wiener measure at 0 on the Poincaré disc.

Let $(X, \mathscr{L})$ be a hyperbolic Riemann surface lamination endowed with the leafwise Poincaré metric $g_{P}$. Recall from Introduction that $\Omega:=\Omega(X, \mathscr{L})$ is the space consisting of all continuous paths $\omega:[0, \infty) \rightarrow X$ with image fully contained in a single leaf. This space is called the sample-path space associated to $(X, \mathscr{L})$. Observe that $\Omega$ can be thought of as the set of all possible paths that a Brownian particle, located at $\omega(0)$ at time $t=0$, might follow as time progresses. For each $x \in X$, let $\Omega_{x}=\Omega_{x}(X, \mathscr{L})$ be the space of all continuous leafwise paths starting at $x$ in $(X, \mathscr{L})$, that is,

$$
\Omega_{x}:=\{\omega \in \Omega: \omega(0)=x\} .
$$

For each $x \in X$, the following mapping

$$
\Omega_{0} \ni \omega \mapsto \phi_{x} \circ \omega \text { maps } \Omega_{0} \text { bijectively onto } \Omega_{x} \text {, }
$$

where $\phi_{x}: \mathbb{D} \rightarrow L_{x}$ is given in (1). Using this bijection we obtain a natural $\sigma$-algebra $\mathscr{A}_{x}$ on the space $\Omega_{x}$, and a natural probability (Wiener) measure $W_{x}$ on $\mathscr{A}_{x}$ as follows:

$$
\mathscr{A}_{x}:=\left\{\phi_{x} \circ A: A \in \mathscr{A}_{0}\right\} \quad \text { and } W_{x}\left(\phi_{x} \circ A\right):=W_{0}(A), \quad A \in \mathscr{A}_{0},
$$


where $\phi_{x} \circ A:=\left\{\phi_{x} \circ \omega: \omega \in A\right\} \subset \Omega_{x}$.

For any function $F \in L^{1}\left(\Omega_{x}, \mathscr{A}_{x}, W_{x}\right)$, the expectation of $F$ at $x$ is the number

$$
\mathbb{E}_{x}[F]:=\int_{\Omega_{x}} F(\omega) d W_{x}(\omega) .
$$

It is well-known (see [8, Proposition C.3.8]) that for any measurable bounded function $f$ on $L_{x}$,

$$
\mathbb{E}_{x}[f(\bullet(t))]=\left(D_{t} f\right)(x), \quad t \in \mathbb{R}^{+},
$$

where $f(\bullet(t))$ is the function given by $\Omega \ni \omega \mapsto f(\omega(t))$.

2.4. Specialization and several classes of cocycles. First we recall some notions and results from [26, Section 9.1]. Fix a point $x \in X$ and let $\phi_{x}: \mathbb{D} \rightarrow L=L_{x}$ be the universal covering map given in (1). We focus on the leaf $L$ and consider the following projectivization of $\mathcal{A}$ :

(18) $\mathcal{A}(\omega, t) u:=[\mathcal{A}(\omega, t) \tilde{u}]$ and $\|\mathcal{A}(\omega, t) u\|:=\frac{\|\mathcal{A}(\omega, t) \tilde{u}\|}{\|\tilde{u}\|}$ for $t \in \mathbb{R}^{+}$and $u \in \mathbb{P}^{d-1}(\mathbb{K})$,

where $\tilde{u}$ is any element in $\mathbb{K}^{d} \backslash\{0\}$ such that $u=[\tilde{u}]$. Here $[\cdot]: \mathbb{K}^{d} \backslash\{0\} \rightarrow \mathbb{P}^{d-1}(\mathbb{K})$ is the canonical projection. For each $u \in \mathbb{P}^{d-1}(\mathbb{K})$, consider the function $f_{x, u}: \mathbb{D} \rightarrow \mathbb{R}$ defined by

$$
f_{x, u}(\zeta):=\log \left\|\mathcal{A}\left(\phi_{x} \circ \omega, 1\right) u\right\|, \quad \zeta \in \mathbb{D},
$$

where $\omega \in \Omega_{0}$ is any path such that $\omega(1)=\zeta$. This definition is well-defined because of the homotopy law for $\mathcal{A}$ (see Definition 1.1) and of the simple connectedness of $\mathbb{D}$. Following [26], $f_{x, u}$ is said to be the specialization of $\mathcal{A}$ at $(x, u)$.

By [26, identities (9.5) and (9.8)], we have that

$$
f_{x, u}(0)=0 \quad \text { and } \quad \mathbb{E}_{x}[\log \|\mathcal{A}(\bullet, t) u\|]=\left(D_{t} f_{x, u}\right)(0), \quad t \in \mathbb{R}^{+},
$$

where $\left(D_{t}\right)_{t \in \mathbb{R}^{+}}$is the family of diffusion operators associated with $\left(\mathbb{D}, g_{P}\right)$.

Next, we recall from [26] two conversion rules for changing specializations in the same leaf. For this purpose let $y \in L$ and pick $\eta \in \phi_{x}^{-1}(y)$. Define $v:=\left[\mathcal{A}\left(\phi_{x} \circ \omega, 1\right) u\right]$, where $\omega \in \Omega_{0}$ is a leafwise path with $\omega(1)=\eta$. As a consequence of the multiplicative law in Definition 1.1, the first conversion rule (see [26, identity (9.6)]) states that

$$
f_{y, v}(\zeta)=f_{x, u}(\zeta)-f_{x, u}(\eta), \quad \zeta \in \mathbb{D} .
$$

We deduce from (20)-(21) and the identity $D_{p} 1=1$ in (11) the following second conversion rule (see [26, identity (9.9)])

$$
\mathbb{E}_{y}[\log \|\mathcal{A}(\bullet, t) v\|]=\left(D_{t} f_{x, u}\right)(\eta)-f_{x, u}(\eta) .
$$

Let $\Delta$ be the Laplacian on the Poincaré disc $\left(\mathbb{D}, g_{P}\right)$, that is, for every function $f \in$ $\mathscr{C}^{2}(\mathbb{D})$,

$$
(\Delta f) g_{P}=i \partial \bar{\partial} f \quad \text { on } \mathbb{D} \text {. }
$$

For every function $f \in \mathscr{C}^{1}(\mathbb{D})$, let $|d f|_{P}$ be the length of the differential $d f$ with respect to $g_{P}$, that is, $|d f|_{P}=|d f| \cdot g_{P}^{-1 / 2}$ on $\mathbb{D}$, where $|d f|$ denotes the Euclidean norm of $d f$. Let dist ${ }_{P}$ denote the Poincaré distance on $\left(\mathbb{D}, g_{P}\right)$. Inspired by Definition 8.3 and 8.4 in Candel [7], we have the following 
Definition 2.7. Let $h$ be a real-valued function defined on $\mathbb{D}$ and let $c, \alpha>0$.

- $h$ is called moderate with constant $c$ if

$$
\log |h(y)-h(z)| \leq c \operatorname{dist}_{P}(y, z)+c, \quad y, z \in \mathbb{D} .
$$

- $h$ is called Hölder of order $\alpha$ with constant $c$ if

$$
|h(y)-h(z)| \leq c\left(\operatorname{dist}_{P}(y, z)\right)^{\alpha}+c, \quad y, z \in \mathbb{D} .
$$

- $h$ is called Lipschitz with constant $c$ if it is Hölder of order 1 with constant $c$.

Notice that our definition of Hölder functions is different from the classical one since we are only concerned about the quotient $|h(y)-h(z)| /\left(\operatorname{dist}_{P}(y, z)\right)^{\alpha}$ when $\operatorname{dist}_{P}(y, z)$ is large enough.

Now we are in the position to formulate new classes of cocycles.

Definition 2.8. Let $\mathcal{A}$ be a cocycle. For every $(x, u) \in X \times \mathbb{P}^{d-1}(\mathbb{K})$, let $f_{x, u}$ denote, as usual, the specialization of $\mathcal{A}$ at $(x, u)$.

- $\mathcal{A}$ is called moderate if there is a constant $c>0$ such that for every $(x, u) \in X \times$ $\mathbb{P}^{d-1}(\mathbb{K}), f_{x, u}$ is a moderate function with constant $c$.

- A moderate cocycle $\mathcal{A}$ is called strongly moderate if it is leafwise $\mathscr{C}^{2}$-differentiable cocycle and if there is a constant $c>0$ such that for every $(x, u) \in X \times \mathbb{P}^{d-1}(\mathbb{K}),\left|\Delta f_{x, u}\right| \leq$ $c$ on $\mathbb{D}$.

- $\mathcal{A}$ is called Hölder if there is $\alpha>0$ such that for every $(x, u) \in X \times \mathbb{P}^{d-1}(\mathbb{K}), f_{x, u}$ is a Hölder function of order $\alpha$. In this context we also say that $\mathcal{A}$ is Hölder of order $\alpha$. If, moreover, there is a constant $c>0$ such that for every $(x, u) \in X \times \mathbb{P}^{d-1}(\mathbb{K}), f_{x, u}$ is a Hölder function of order $\alpha$ with constant $c$, then we say that $\mathcal{A}$ is uniformly Hölder (of order $\alpha$ ).

- $\mathcal{A}$ is called Lipschitz (resp. uniformly Lipschitz) if $\mathcal{A}$ is Hölder (resp. uniformly Hölder) of order 1.

Remark 2.9. (1) As an immediate consequence of Definition 2.8, the class of Hölder (resp. uniformly Hölder) cocycles $\mathcal{A}$ of order $\alpha$ is increasing in $\alpha$.

(2) It is worthy noting the following difference between a moderate cocycle and a Hölder one. For a moderate cocycle, each specialization $f_{x, u}$ is a moderate function with the same constant $c$; whereas for a Hölder cocycle of order $\alpha$, each specialization $f_{x, u}$ is a Hölder function of order $\alpha$ with some constant $c_{x, u}$ which depends on $x$ and $u$. So a moderate cocycle need not to be Hölder, and vice versa. Clearly, every uniformly Hölder cocycle of order $\alpha$ is Hölder of order $\alpha$. However, using Definition 2.7 and Definition 2.8, it can be checked that a uniformly Hölder cocycle is moderate. As a partial converse of the last fact, it is shown in Lemma 5.2 below that a strongly moderate cocycle is necessarily uniformly Lipschitz.

(3) Using formula (21), Definition 2.8 reduces to asking the desired properties of $f_{x, u}$ for only one point $x$ in each leaf $L$ of the lamination.

Strongly moderate and uniformly Hölder cocycles exist in abundance. Here is a simple sufficient criterion.

Proposition 2.10. A $\mathscr{C}^{2}$-differentiable cocycle $\mathcal{A}$ on a hyperbolic Riemann surface lamination $(X, \mathscr{L})$ with $X$ compact is both strongly moderate and uniformly Lipschitz. 
Proof. Since $X$ is compact, we know from Candel [6] that $g_{p}$ is transversally continuous. This, coupled with the assumption that $\mathcal{A}$ is $\mathscr{C}^{2}$-differentiable, equality (19) and formula (21), implies that

$$
\left|d f_{x, u}\right|_{P} \leq c \text { and }\left|\Delta f_{x, u}\right| \leq c \text { for a constant } c>0 \text { independent of } x \text { and } u .
$$

The bound on $\left|d f_{x, u}\right|_{P}$ yields that $\mathcal{A}$ is uniformly Lipschitz, hence moderate by Item 2 . in Remark 2.9. This, coupled with the bound on $\Delta f_{x, u}$ implies that $\mathcal{A}$ is strongly moderate.

Example 2.11. Let $(M, \mathscr{L}, E)$ be a transversally $\mathscr{C}^{2}$-smooth (resp. transversally holomorphic) singular foliation by hyperbolic Riemann surfaces with the set of singularities $E$ in a Riemannian manifold (resp. Hermitian complex manifold) $M$. Consider a leafwise saturated, compact set $X \subset M \backslash E$. So the restriction of the foliation $(M \backslash E, \mathscr{L})$ to $X$ gives an inherited lamination $(X, \mathscr{L})$. Moreover, the holonomy cocycle of $(M \backslash E, \mathscr{L})$ induces, by restriction, an inherited $\mathscr{C}^{2}$-differentiable cocycle $\mathcal{A}$ on $(X, \mathscr{L})$. By Proposition 2.10, $\mathcal{A}$ is strongly moderate and uniformly Lipschitz.

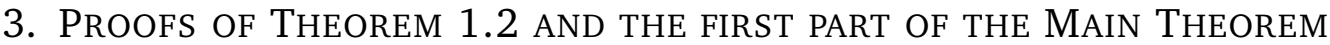

We keep the hypotheses and notation of Theorem 1.2. In what follows, let $\mathcal{A}^{+}:=\mathcal{A}$ and $\mathcal{A}^{-}:=\mathcal{A}^{-1}$, and write $\mathcal{A}^{ \pm}$for both $\mathcal{A}^{+}$and $\mathcal{A}^{-}$.

Lemma 3.1. For every $t \in \mathbb{R}^{+}$there is a constant $c=c_{t}>0$ such that

$$
\int_{\Omega_{x}} \sup _{0 \leq s \leq t}\left|\log \left\|\mathcal{A}^{ \pm}(\omega, s)\right\|\right| d W_{x}(\omega) \leq c \quad \text { for all } x \in X .
$$

Proof. Assume without loss of generality that $t=1$ and let $x \in X$. Since $\mathcal{A}$ is moderate, there exists a constant $c^{\prime}>0$ independent of $x$ such that, for every universal covering map $\phi_{x}: \mathbb{D} \rightarrow L_{x}$ given in (1), we have that

$$
\left|\log \left\|\mathcal{A}^{ \pm 1}\left(\phi_{x} \circ \omega, s\right)\right\|\right| \leq \exp \left(c^{\prime}+c^{\prime} \operatorname{dist}_{P}(\omega(s), \omega(0))\right), \quad \omega \in \Omega_{0}, s \in \mathbb{R}^{+} .
$$

Recall from (14) and (15) that $\Omega_{0} \ni \omega \mapsto \phi_{x} \circ \omega \in \Omega_{x}$ is a bijection that induces $W_{x}$ from $W_{0}$. So

$$
\begin{aligned}
\int_{\Omega_{x}} \sup _{0 \leq s \leq 1}\left|\log \left\|\mathcal{A}^{ \pm}(\omega, s)\right\|\right| d W_{x}(\omega) & \leq \int_{\Omega_{0}} \exp \left(c^{\prime}+c^{\prime} \cdot \sup _{0 \leq s \leq 1} \operatorname{dist}_{P}(\omega(s), \omega(0))\right) d W_{0}(\omega) \\
& =e^{c^{\prime}} \int_{\Omega_{0}} \exp \left(c^{\prime} \cdot \sup _{0 \leq s \leq 1} \operatorname{dist}_{P}(\omega(s), \omega(0))\right) d W_{0}(\omega) .
\end{aligned}
$$

Moreover, for every positive-valued function $f \in L^{1}\left(\Omega_{0}, W_{0}\right)$ we have by Fubini's theorem that

$$
\int_{0}^{\infty} W_{0}\{f(\omega)>r\} d r=\int_{\Omega_{0}} f(\omega) d W_{0}(\omega)
$$

Applying the above identity to

$$
f(\omega):=\exp \left(c^{\prime} \cdot \sup _{0 \leq s \leq 1} \operatorname{dist}_{P}(\omega(s), \omega(0))\right) \text { for } \omega \in \Omega_{0},
$$


it follows that the right hand side of (23) is equal to

$$
e^{c^{\prime}} \int_{0}^{\infty} W_{0}\left\{\omega \in \Omega_{0}: \exp \left(c^{\prime} \cdot \sup _{0 \leq s \leq 1} \operatorname{dist}_{P}(\omega(s), \omega(0))\right)>r\right\} d r .
$$

On the other hand, combining Lemma 8.16 and Corollary 8.8 in [7], we can show that there is a constant $c^{\prime \prime}>0$ such that for all $r \geq 1$,

$$
W_{0}\left\{\omega \in \Omega_{0}: \sup _{s \in[0,1]} \operatorname{dist}_{P}(\omega(s), \omega(0))>r\right\}<c^{\prime \prime} e^{-r^{2} / 64} .
$$

This implies that the integral in (24) is dominated by

$$
\begin{aligned}
& 1+\int_{1}^{\infty} W_{0}\left\{\omega \in \Omega_{0}: \exp \left(c^{\prime} \cdot \sup _{0 \leq s \leq 1} \operatorname{dist}_{P}(\omega(s), \omega(0))\right)>r\right\} d r \\
= & \left.1+\int_{1}^{\infty} W_{0}\left\{\omega \in \Omega_{0}: \sup _{0 \leq s \leq 1} \operatorname{dist}_{P}(\omega(s), \omega(0))\right)>\frac{\ln r}{c^{\prime}}\right\} d r \\
< & 1+c^{\prime \prime} \int_{1}^{\infty} e^{-\left(\ln r / 8 c^{\prime}\right)^{2}} d r<\infty .
\end{aligned}
$$

This, coupled with (23), completes the proof.

Remark 3.2. The proof of the upper bound of the right hand side of (23) also shows that for $c^{\prime}, t>0$,

$$
\int_{\Omega_{0}} \exp \left(c^{\prime}+c^{\prime} \cdot \operatorname{dist}_{P}(\omega(t), \omega(0))\right) d W_{0}(\omega)<\infty .
$$

Now we arrive at the

End of the proof of Theorem 1.2. Since $\mu$ is a finite measure, Lemma 3.1, applied to $t=1$, gives that

$$
\int_{x \in X}\left(\int_{\Omega_{x}} \sup _{0 \leq s \leq 1}\left|\log \left\|\mathcal{A}^{ \pm}(\omega, s)\right\|\right| d W_{x}(\omega)\right) d \mu(x)<\infty .
$$

So $\mathcal{A}$ satisfies the integrability condition stated in [26, Theorem 3.7]. On the other hand, by Theorem 2.6 (iii), we know that $\mu$ is ergodic. Consequently, we may apply [26, Corollary 3.8 and Theorem 7.3] and the theorem follows.

To prove the first part of the Main Theorem, we need the following result on Brownian motion on the Poincaré disc $\left(\mathbb{D}, g_{P}\right)$.

Lemma 3.3. (i) For $W_{0}$-almost every $\omega \in \Omega_{0}$, the limit $\omega(\infty):=\lim _{t \rightarrow \infty} \omega(t)$ exists and is a point in $\partial \mathbb{D}$. In this case let $\theta=\theta_{\omega}$ be the unique number in $[0,1)$ such that $e^{2 \pi i \theta}=\omega(\infty)$, and denote by $\gamma_{\omega}$ the unit-speed geodesic ray which is the radius of $\mathbb{D}$ issued from 0 and landing at $\omega(\infty)$.

(ii) For every Borel set $B \subset \partial \mathbb{D}$, we have that

$$
W_{0}\left(\left\{\omega \in \Omega_{0}: \exists \omega(\infty) \in B\right\}\right)=\operatorname{Leb}\left\{\theta \in[0,1): e^{2 \pi i \theta} \in B\right\} .
$$

Here Leb denotes the Lebesgue measure on $[0,1)$.

(iii) Let $\rho>1$. Then, for $W_{0}$-almost every $\omega \in \Omega_{0}$, there is a constant $c_{\omega}>0$ such that

$$
\operatorname{dist}_{P}\left(\omega(t), \gamma_{\omega}(t)\right) \leq c_{\omega} t^{1 / 2}(\log t)^{\rho} \quad \text { for } t>2 .
$$


Roughly speaking, assertion (i) says that Brownian trajectories issued from 0 on the Poincaré disc are shadowed by the unit-speed geodesic rays which are radii of $\mathbb{D}$. Assertion (ii) states that these radii are uniformly distributed with respect to the normalized rotation measure on $\partial \mathbb{D}$. Moreover, assertion (iii) quantifies the distance, as the time progresses, between a Brownian trajectory and its corresponding unit-speed geodesic ray.

Proof. Assertion (i) and (ii) are classical, see, for example, Ancona's work [2, Section 7].

To prove assertion (iii) recall from [2, Theorem 7.3] that for $W_{0}$-almost every $w \in \Omega_{0}$, there is a constant $c_{\omega}^{\prime}>0$ such that

$$
\operatorname{dist}_{P}\left(\omega(t), \gamma_{\omega}\left(\mathbb{R}^{+}\right)\right) \leq c_{\omega}^{\prime} \log t \quad \text { for } t>2 .
$$

Since the point $\gamma_{\omega}(s)$ with $s=s_{\omega, t}:=\operatorname{dist}(\omega(t), 0)$ is the nearest point in $\gamma_{\omega}\left(\mathbb{R}^{+}\right)$with respect to the point $\omega(t)$, it follows that

$$
\operatorname{dist}_{P}\left(\omega(t), \gamma_{\omega}(s)\right) \leq c_{\omega}^{\prime} \log t \quad \text { for } t>2 .
$$

On the other hand, recall from Lyons' work [24, pp. 3-4] that for $W_{0}$-almost every $w \in \Omega_{0}$, there is a constant $c_{\omega}^{\prime}>0$ such that

$$
\left|\operatorname{dist}_{P}(\omega(t), 0)-t\right| \leq c_{\omega}^{\prime} t^{1 / 2}(\log t)^{\rho} \quad \text { for } t>2 .
$$

It is worthy noting that Lyons' estimate relies on a previous result of Cranston [11] on the boundary behavior of Brownian trajectories. This, combined with the previous estimate, implies that

$$
\begin{aligned}
\operatorname{dist}_{P}\left(\omega(t), \gamma_{\omega}(t)\right) & \leq \operatorname{dist}_{P}\left(\omega(t), \gamma_{\omega}(s)\right)+\operatorname{dist}_{P}\left(\gamma_{\omega}(t), \gamma_{\omega}(s)\right) \\
& =\operatorname{dist}_{P}\left(\omega(t), \gamma_{\omega}(s)\right)+\left|\operatorname{dist}_{P}(\omega(t), 0)-t\right| \\
& \leq c_{\omega}^{\prime} \log t+c_{\omega}^{\prime} t^{1 / 2}(\log t)^{\rho} \quad \text { for } t>2 .
\end{aligned}
$$

Choosing $c_{\omega}:=c_{\omega}^{\prime}\left(1+(\log 2)^{1-\rho}\right)$, assertion (iii) follows.

Now we are in the position to prove the short part of the Main Theorem. In fact, we are partly inspired by the proof of [13, Lemma 6.12], where Deroin and Dupont investigate a particular cocycle of rank 1. But in their context, their method is only applicable for Lipschitz cocycles.

Proof of assertion (i) of Theorem 1.4. We only give the proof of equality (7) since equality (8) can be demonstrated in the same way. The assumption of the theorem allows us to apply Theorem 1.2 to the cocycle $\mathcal{A}$. Consequently, we obtain properties (i) and (ii) of Theorem 1.2. In the rest of the proof we keep the notation $Y, m, V_{i}(x), \chi_{i}$ introduced in Theorem 1.2. Fix an integer $1 \leq i_{0} \leq m$ and fix a point $x_{0} \in Y$. Let $v$ be an arbitrary vector in $V_{i_{0}}\left(x_{0}\right) \backslash V_{i_{0}+1}\left(x_{0}\right)$. We need to show that

$$
\lim _{R \rightarrow \infty} \mathscr{E}\left(x_{0}, \theta, R, v\right)=\chi_{i_{0}} \quad \text { for Leb-almost every } \theta \in[0,1) .
$$

Let $\phi=\phi_{x_{0}}: \mathbb{D} \rightarrow L:=L_{x_{0}}$ be the universal covering map given in (1). Fix $\rho>1$. Let $\omega$ be a generic path (in the sense of the measure $W_{0}$ ) in $\Omega_{0}$. So $\omega$ satisfies Lemma 3.3 (i) and (iii). Writing $\gamma:=\gamma_{\omega}$, we get a constant $c_{\omega}$ such that

$$
\operatorname{dist}_{P}(\omega(R), \gamma(R)) \leq c_{\omega} R^{1 / 2}(\log R)^{\rho} \quad \text { for } R>2 .
$$


On the other hand, by (3), we also have that

$$
\lim _{R \rightarrow \infty} \frac{1}{R} \log \frac{\|\mathcal{A}(\phi \circ \omega, R) v\|}{\|v\|}=\chi_{i_{0}} .
$$

For every $R>2$ let $x_{R}:=\omega(R)$ and $y_{R}:=\gamma(R)$. By (19), we have that

$$
\frac{1}{R} \log \frac{\|\mathcal{A}(\phi \circ \omega, R) v\|}{\|v\|}-\frac{1}{R} \log \frac{\|\mathcal{A}(\phi \circ \gamma, R) v\|}{\|v\|}=\frac{1}{R}\left(f_{x_{0},[v]}\left(x_{R}\right)-f_{x_{0},[v]}\left(y_{R}\right)\right) .
$$

Since $\mathcal{A}$ is Hölder of order $\alpha<2$, there is a constant $c$ depending only on $\mathcal{A}$ and $\left(x_{0}, v\right)$ such that the modulus of the right hand side of (28) is bounded by

$$
R^{-1}\left(c\left(\operatorname{dist}_{P}\left(x_{R}, y_{R}\right)\right)^{\alpha}+c\right) \text {. }
$$

On the other hand, by (26), we know that $\operatorname{dist}_{P}\left(x_{R}, y_{R}\right)=\operatorname{dist}_{P}(\omega(R), \gamma(R)) \leq c_{\omega} R^{1 / 2}(\log R)^{\rho}$. Consequently, using that $\alpha<2$, it follows that the right hand side of (28) tends to 0 as $R \rightarrow \infty$. This, combined with (28) and (27), implies that

$$
\lim _{R \rightarrow \infty} \frac{1}{R} \log \frac{\|\mathcal{A}(\phi \circ \gamma, R) v\|}{\|v\|}=\chi_{i_{0}}, \quad \text { for } W_{0} \text {-almost every } \omega \in \Omega_{0} .
$$

Since $\phi \circ \gamma=\gamma_{x_{0}, \theta}$, where $\theta:=\theta_{\omega}$ (see Lemma 3.3) and $\gamma_{x_{0}, \theta}$ is given in (6), the last equality may be rewritten as

$$
\lim _{R \rightarrow \infty} \frac{1}{R} \log \frac{\left\|\mathcal{A}\left(\gamma_{x_{0}, \theta}, R\right) v\right\|}{\|v\|}=\chi_{i_{0}}, \quad \text { for } W_{0} \text {-almost every } \omega \in \Omega_{0} \text { and for } \theta:=\theta_{\omega} .
$$

Putting this together with Lemma 3.3 (ii), (25) follows. The proof of Part (i) of the Main Theorem is thereby completed.

\section{EXPECTATION CONVERGENCE}

4.1. Statement of the expectation convergence and a reduction. The main purpose of this section is to prove the following expectation convergence which is a key ingredient in the proof of the second part of the Main Theorem.

Theorem 4.1. Let $(X, \mathscr{L})$ be a hyperbolic Riemann surface lamination and $T$ a harmonic current. Let $\mu$ be the measure associated to $T$ by (2). Assume, moreover, that $T$ is extremal and that $\mu$ is a harmonic measure. Consider a moderate cocycle $\mathcal{A}: \Omega \times \mathbb{R}^{+} \rightarrow \operatorname{GL}(d, \mathbb{K})$. Let $Y$ be a leafwise saturated Borel set of full T-measure which satisfies the conclusion of Theorem 1.2 Let $\mathbb{K}^{d}=\oplus_{i=1}^{m} H_{i}(x), x \in Y$, be the Oseledec decomposition given by Theorem 1.2 and $\chi_{m}<\chi_{m-1}<\cdots<\chi_{2}<\chi_{1}$ the corresponding Lyapunov exponents. For each $1 \leq i \leq m$ and $n \in \mathbb{N} \backslash\{0\}$, define two maximal and minimal functions $\mathbf{M}_{i, n}, \mathbf{m}_{i, n}: Y \rightarrow$ $[-\infty, \infty]$ by

$$
\begin{aligned}
& \mathbf{M}_{i, n}(x):=\sup _{v \in H_{i}(x) \backslash\{0\}} \frac{1}{n} \mathbb{E}_{x}\left[\log \frac{\|\mathcal{A}(\bullet, n) v\|}{\|v\|}\right], \quad x \in Y ; \\
& \mathbf{m}_{i, n}(x):=\inf _{v \in H_{i}(x) \backslash\{0\}} \frac{1}{n} \mathbb{E}_{x}\left[\log \frac{\|\mathcal{A}(\bullet, n) v\|}{\|v\|}\right], \quad x \in Y,
\end{aligned}
$$

where $\log \frac{\|\mathcal{A}(\bullet, n) v\|}{\|v\|}$ denotes the function

$$
\Omega_{x} \ni \omega \mapsto \log \frac{\|\mathcal{A}(\omega, n) v\|}{\|v\|} .
$$


Then there is a Borel set $Y_{0} \subset Y$ of full T-measure such that

$$
\lim _{n \rightarrow \infty}\left[\mathbf{m}_{i, n}(x), \mathbf{M}_{i, n}(x)\right]=\chi_{i}, \quad x \in Y_{0} \quad \text { and } \quad 1 \leq i \leq m .
$$

The rest of the section is devoted to the proof of Theorem 4.1 ,

We make the following reduction. Fix an index $i_{0}: 1 \leq i_{0} \leq m$. Choose measurable maps $\psi_{1}, \ldots, \psi_{d_{i_{0}}}: X \rightarrow \mathbb{K}^{d}$ such that for all $x \in Y,\left\{\psi_{1}(x), \ldots, \psi_{d_{i_{0}}}(x)\right\}$ is an orthonormal basis of $H_{i_{0}}(x)$. Consider the cocycle $\mathcal{B}: \Omega \times \mathbb{R}^{+} \rightarrow \mathrm{GL}\left(d_{i_{0}}, \mathbb{K}\right)$ defined by

$$
\mathcal{B}(\omega, t) v:=\psi_{y}^{-1}\left(\mathcal{A}(\omega, t)\left(\psi_{x}(v)\right)\right), \quad v \in \mathbb{K}^{d_{i_{0}}},
$$

where $x:=\omega(0), y:=\omega(t)$, and for each $x \in Y, \psi_{x}: \mathbb{K}^{d_{i_{0}}} \rightarrow H_{i_{0}}(x)$ is the $\mathbb{K}$-linear isomorphism given by

$$
\psi_{x}(v):=\sum_{j=1}^{d_{i_{0}}} \lambda_{j} \psi_{j}(x), \quad \text { for } v:=\left(\lambda_{1}, \ldots, \lambda_{d_{i_{0}}}\right) \in \mathbb{K}^{d_{i_{0}}} .
$$

Since $\psi_{x}$ preserves the Euclidean norms, we infer that the specialization of $\mathcal{A}$ at $(x, u)$ for any $x \in Y$ and $u \in H_{i_{0}}(x)$ is the same as the specialization of $\mathcal{B}$ at $\left(x, \psi_{x}^{-1}(u)\right)$. Consequently, the proof of Theorem 4.1 for the cocycle $\mathcal{A}$ and $i=i_{0}$ reduces to the proof for the cocycle $\mathcal{B}$ having the unique Lyapunov exponent $\chi_{i_{0}}$. Therefore, in the rest of this section, we may assume without loss of generality that

The cocycle $\mathcal{A}$ possesses a unique Lyapunov exponent $\chi$ (that is, $m=1$ ). Moreover, we will write $\mathbf{M}_{n}$ (resp. $\mathbf{m}_{n}$ ) instead of the unique maximal function $\mathbf{M}_{1, n}$ (resp. the unique minimal function $\mathbf{m}_{1, n}$ ).

4.2. Ledrappier type characterization of Lyapunov spectrum. We recall from [26, Section 9.2] some results about dual spaces (see also [31]). Let $(X, \mathscr{B}(X), \mu)$ be a probability Borel space, where $X$ is a Hausdorff topological space. Let $E$ be a separable Banach space with dual space $E^{*}$ and let $\langle\cdot, \cdot\rangle$ denote the pairing between $E$ and $E^{*}$. Let $L_{\mu}^{1}(E)$ be the space of all $\mu$-measurable functions $f: X \rightarrow E$ such that $\|f\|:=\int_{X}\|f(x)\| d \mu(x)<\infty$. This is a Banach space with the norm $f \mapsto\|f\|$, where two functions $f$ and $g$ are identified if $f=g \mu$-almost everywhere. Let $L_{\mu}^{\infty}\left(E^{*}, E\right)$ be the space of all maps $f: X \rightarrow E^{*}$ for which the function $X \ni x \mapsto\langle f(x), v\rangle$ is bounded and measurable for each $v \in E$, where two such functions $f, g$ are identified if $X \ni x \mapsto\langle f(x), v\rangle$ and $X \ni x \mapsto\langle g(x), v\rangle$ are equal $\mu$-almost everywhere for every $v \in E$. This is a Banach space with the norm

$$
\|f\|_{\infty}:=\operatorname{ess} . \sup _{x \in X}\|f(x)\|=\inf _{Y \in \mathscr{B}(X): \mu(Y)=1} \sup _{x \in Y}\|f(x)\|,
$$

which is finite by the principle of uniform boundedness. Consider the map $\Lambda: L_{\mu}^{\infty}\left(E^{*}, E\right) \rightarrow$ $\left(L_{\mu}^{1}(E)\right)^{*}$, given by

$$
(\Lambda \gamma)(f):=\int_{X}\langle\gamma(x), f(x)\rangle d \mu(x)
$$

where the map $\gamma: X \rightarrow E^{*}$ is in $L_{\mu}^{\infty}\left(E^{*}, E\right)$, and the map $f: X \rightarrow E$ is in $L_{\mu}^{1}(E)$. By [5], $\Lambda$ is an isomorphism of Banach spaces. In what follows, for a locally compact metric space $\Sigma$, we denote by $\mathscr{M}(\Sigma)$ the space of all Radon measures on $\Sigma$ with mass $\leq 1$. 
We will be interested in the case where $E:=\mathscr{C}(P, \mathbb{R})$ for a compact metric space $P$. So $\mathscr{M}(P)$ is the closed unit ball of $E^{*}$. The set $L_{\mu}^{\infty}(\mathscr{M}(P))$ of all measurable maps $\gamma: X \rightarrow \mathscr{M}(P)$ is contained in the unit ball of $L_{\mu}^{\infty}\left(E^{*}, E\right)$, and is closed with respect to the weak-star topology $L_{\mu}^{\infty}\left(E^{*}, E\right)$. Hence, $L_{\mu}^{\infty}(\mathscr{M}(P))$ is compact with respect to this topology. The set $L_{\mu}^{\infty}(\mathscr{M}(P))$ can be identified with a subset of the following space:

$$
\mathscr{M}_{\mu}(X \times P):=\{\lambda \in \mathscr{M}(X \times P): \lambda \text { projects to } \mu \text { on } X\} .
$$

via the map $L_{\mu}^{\infty}(\mathscr{M}(P)) \ni \nu \mapsto \lambda \in \mathscr{M}(X \times P)$, where for $f \in L_{\mu}^{1}(\mathscr{C}(P, \mathbb{R}))$, we have

$$
\int_{X \times P}\langle f(x), u\rangle d \lambda(x, u)=\int_{X}\langle f(x), \nu(x)\rangle d \mu(x) .
$$

Here, $\langle f(x), u\rangle$ denotes the evaluation of the function $f(x) \in \mathscr{C}(P, \mathbb{R})$ at the point $u \in P$, and $\langle f(x), \nu(x)\rangle$ denotes the pairing between $E$ and $E^{*}$ evaluated at $f(x) \in E$ and $\nu(x) \in$ $E^{*}$.

In the remaining part of the section, let $(X, \mathscr{L})$ be a hyperbolic Riemann lamination endowed with a harmonic probability measure $\mu$ which is ergodic, let $\mathcal{A}: \Omega(X, \mathscr{L}) \times$ $\mathbb{R}^{+} \rightarrow \mathrm{GL}(d, \mathbb{K})$ be a cocycle admitting a unique Lyapunov exponent $\chi$ with respect to $\mu$. Assume in addition that $\mathcal{A}$ is moderate. Set

$$
P=\mathbb{P}:=\mathbb{P}^{d-1}(\mathbb{K}) \quad \text { and } \quad \mathscr{C}(\mathbb{P}):=\mathscr{C}(P, \mathbb{R}) .
$$

Consider the cylinder lamination of $\mathcal{A}$, denoted by $\left(X_{\mathcal{A}}, \mathscr{L}_{\mathcal{A}}\right)$, which is defined as follows. The ambient topological space $X_{\mathcal{A}}$ of the cylinder lamination is $X \times \mathbb{P}$ which is independent of $\mathcal{A}$. Its leaves are defined as follows. For a point $(x, u) \in X \times \mathbb{P}$ and for every simply connected plaque $K$ of $(X, \mathscr{L})$ passing through $x$, we define the plaque $\mathcal{K}$ of $\left(X \times \mathbb{P}, \mathscr{L}_{\mathcal{A}}\right)$ passing through $(x, U)$ by

$$
\mathcal{K}=\mathcal{K}(K, x, u):=\left\{(y, \mathcal{A}(\omega, 1) u): y \in K, \omega \in \Omega_{x}, \omega(1)=y, \omega[0,1] \subset K\right\},
$$

where $\mathcal{A}(\omega, 1) u$ is defined using (18).

Note that the projection on the first factor $\mathrm{pr}_{1}: X \times \mathbb{P} \rightarrow X$ maps each leaf of $\left(X_{\mathcal{A}}, \mathscr{L}_{\mathcal{A}}\right)=\left(X \times \mathbb{P}, \mathscr{L}_{\mathcal{A}}\right)$ onto each leaf of $(X, \mathscr{L})$ locally homeomorphically. Therefore, we endow each leaf of the cylinder lamination with the (leafwise) Poincaré metric, still denoted by $g_{P}$. The Laplacian and the one parameter family $\left\{D_{t}: t \geq 0\right\}$ of the diffusion operators are defined using the newly-defined metric $g_{P}$. Since the local expression of $\mathcal{A}$ on flow boxes is, in general, only measurable, the cylinder lamination $\left(X \times \mathbb{P}, \mathscr{L}_{\mathcal{A}}\right)$ is a measurable lamination in the sense of [26, Definition 2.2].

For a positive finite Borel measure $\nu$ on $X_{\mathcal{A}}=X \times \mathbb{P}$ and $t \in \mathbb{R}^{+}, D_{t} \nu$ is the positive finite Borel measure on $X_{\mathcal{A}}$ (unique in the sense of $\nu$-almost everywhere) satisfies the following condition

$$
\int_{X_{\mathcal{A}}} D_{t} f(x, u) d \nu(x, u)=\int_{X_{\mathcal{A}}} f(x, u) d\left(D_{t} \nu\right)(x, u)
$$

for every bounded measurable function $f: X_{\mathcal{A}} \rightarrow \mathbb{R}$. When $\nu$ is a probability measure, so is $D_{t} \nu$ (see, for example, [26, Remark B.14]).

Recall from [26, Lemma 9.6] the following result 
Lemma 4.2. For every $t \geq 0$, the operators $D_{t}: L_{\mu}^{\infty}(\mathscr{M}(\mathbb{P})) \rightarrow L_{\mu}^{\infty}(\mathscr{M}(\mathbb{P}))$ and $D_{t}:$ $L_{\mu}^{1}(\mathscr{C}(\mathbb{P})) \rightarrow L_{\mu}^{1}(\mathscr{C}(\mathbb{P}))$ are contractions, that is, $\left\|D_{t} \nu\right\|_{\infty} \leq\|\nu\|_{\infty}$ for $\nu \in L_{\mu}^{\infty}(\mathscr{M}(\mathbb{P}))$ and $\left\|D_{t} f\right\| \leq\|f\|$ for $f \in L_{\mu}^{1}(\mathscr{C}(\mathbb{P}))$.

Definition 4.3. An element $\nu$ in $L_{\mu}^{\infty}(\mathscr{M}(\mathbb{P}))$ is said to be a $\mathcal{A}$-weakly harmonic measure if

$$
\int_{X_{\mathcal{A}}} D_{1} f d \nu=\int_{X_{\mathcal{A}}} f d \nu
$$

for all functions $f \in L_{\mu}^{1}(\mathscr{C}(\mathbb{P}))$.

Denote by $\operatorname{Har}_{\mu}\left(X_{\mathcal{A}}\right)$ (or equivalently $\operatorname{Har}_{\mu}(X \times \mathbb{P})$ when $\mathcal{A}$ is clear from the context) the set of all $\mathcal{A}$-weakly harmonic elements. This is a convex subset of $L_{\mu}(\mathscr{M}(\mathbb{P}))$. Using Lemma 4.2, this set is also closed.

An element $\nu \in \operatorname{Har}_{\mu}\left(X_{\mathcal{A}}\right)$ is said to be extremal if it is an extremal point of this convex closed cone, that is, if $\nu=t \nu_{1}+(1-t) \nu_{2}$ for some $0<t<1$ and $\nu_{1}, \nu_{2} \in \operatorname{Har}_{\mu}(X \times \mathbb{P})$, then $\nu_{1}$ and $\nu_{2}$ are constants times of $\nu$. Clearly, if $\operatorname{Har}_{\mu}(X \times \mathbb{P}) \neq\{0\}$, the set of its nonzero extremal elements is always nonempty.

Recall from [26, Proposition 9.5] that every extremal element $\nu$ of $\operatorname{Har}_{\mu}(X \times \mathbb{P})$ is also an ergodic measure for the cylinder lamination. In particular, if $\operatorname{Har}_{\mu}(X \times \mathbb{P}) \neq\{0\}$, there always exists a nonzero $\mathcal{A}$-weakly harmonic element which is also ergodic.

Consider the functions $\varphi$ and $\varphi_{n}: X \times \mathbb{P} \rightarrow \mathbb{R}$ given by

$$
\begin{aligned}
\varphi(x, u) & :=\int_{\Omega_{x}} \log \|\mathcal{A}(\omega, 1) u\| d W_{x}(\omega), \\
\varphi_{n} & :=\frac{1}{n} \sum_{i=0}^{n-1} D_{i} \varphi .
\end{aligned}
$$

Lemma 4.4. (i) The function $\varphi$ belongs to $L_{\mu}^{1}(\mathscr{C}(\mathbb{P}))$.

(ii) For every $n \geq 1$,

$$
\varphi_{n}(x, u)=\frac{1}{n} \int_{\Omega_{x}} \log \|\mathcal{A}(\omega, n) u\| d W_{x}(\omega), \quad(x, u) \in X \times \mathbb{P} .
$$

(iii) For every $n \geq 1, \varphi_{n}$ belongs to $L_{\mu}^{1}(\mathscr{C}(\mathbb{P}))$. Moreover, it is a Borel measurable function from $X \times \mathbb{P} \rightarrow \mathbb{R}$.

Proof. Observe that for each $\omega \in \Omega_{x}$, the map $\mathbb{P} \ni u \mapsto \log \|\mathcal{A}(\omega, 1) u\|$ is continuous. Moreover,

$$
\left|\log \left\|\mathcal{A}^{ \pm}(\omega, 1) u\right\|\right| \leq\left|\log \left\|\mathcal{A}^{ \pm}(\omega, 1)\right\|\right|, \quad u \in \mathbb{P} .
$$

On the other hand, by Lemma 3.1 we get a constant $c<0$ such that

$$
\int_{\Omega_{x}}|\log \|\mathcal{A}(\omega, 1)\|| d W_{x}(\omega)<c, \quad x \in X .
$$

Putting these together and using that $\mu$ is a finite measure, we may apply the Lebesgue's dominated convergence. Consequently, $\varphi(x, \cdot)$ is continuous on $\mathbb{P}$ and $\|\varphi(x, \cdot)\|_{\mathscr{C}(\mathbb{P})} \leq c$ for any point $x \in X$. This proves assertion (i).

Using assertion (i), we proceed as in the proof of [26, Lemma 9.9]. Assertion (ii) follows. 
Arguing as in the proof of assertion (i) and using assertion (ii) yield the first part of assertion (iii). The second one is an immediate consequence of assertion (ii) (see also [26, Theorem 2.6 (i) and Appendix A.7]).

The following result has been proved in [26, Lemma 9.10].

Lemma 4.5. Let $\left(\nu_{n}\right)_{n=1}^{\infty} \subset L_{\mu}^{\infty}(\mathscr{M}(\mathbb{P}))$ be such that for each $n \geq 1$ and for $\mu$-almost every $x \in X, \nu_{n}(x)$ is a Dirac mass at some point $u_{n}(x) \in \mathbb{P}$.

(i) Then there is a subsequence $\left(\nu_{n_{j}}\right)_{j=1}^{\infty}$ such that $\frac{1}{n_{j}} \sum_{k=0}^{n_{j}-1} D_{k} \nu_{n_{j}}$ converges weakly to a probability measure $\nu \in \operatorname{Har}_{\mu}(X \times \mathbb{P})$. In particular, there always exists a probability ergodic $\mathcal{A}$-weakly harmonic measure on $X \times \mathbb{P}$.

(ii) Moreover, we have that

$$
\lim _{j \rightarrow \infty} \int_{X} \varphi_{n_{j}}\left(x, u_{n_{j}}(x)\right) d \mu(x)=\int_{X \times \mathbb{P}} \varphi d \nu
$$

where $\varphi_{n_{j}}$ and $\varphi$ are given by (31).

The last result of the subsection provides an integral formula for the Lyapunov exponent $\chi$.

Theorem 4.6. For every probability measure $\nu \in \operatorname{Har}_{\mu}(X \times \mathbb{P})$ we have that $\int_{X \times \mathbb{P}} \varphi d \nu=\chi$.

Proof. Consider first the case where $\nu$ is ergodic. Since $\mathcal{A}$ admits a unique Lyapunov exponent $\chi$, the theorem follows from [26, Theorem 9.22, Part 1) (i)].

Now consider the general case. The Choquet unique representation theorem (see, for example, [8, Theorem 2.6.23]) provides the integral representation of $\nu$ as a convex combinations of elements of $K$, where $K$ denotes all extremal elements of the closed convex cone $\operatorname{Har}_{\mu}(X \times \mathbb{P})$ :

$$
\nu=\int_{K} \tau d \rho(\tau)
$$

where $\rho$ is a probability measure on $K$. Therefore, we get that

$$
\int_{X \times \mathbb{P}} \varphi d \nu=\int_{\tau \in K}\left(\int_{X \times \mathbb{P}} \varphi d \tau\right) d \rho(\tau)
$$

On the other hand, since $\tau \in K$ is ergodic, the first case implies that the inner integral on the right hand side is equal to $\chi$. Hence, the right hand side is also equal to $\chi$. This completes the proof.

\subsection{Proof of the expectation convergence.}

Lemma 4.7. (i) For each $n \geq 1$,

$$
\mathbf{M}_{n}(x)=\sup _{u \in \mathbb{P}} \varphi_{n}(x, u) \quad \text { and } \quad \mathbf{m}_{n}(x):=\inf _{u \in \mathbb{P}} \varphi_{n}(x, u), \quad x \in X .
$$

(ii) For each $n \geq 1, \mathbf{M}_{n}$ and $\mathbf{m}_{n}$ are Borel functions and $\mu$-integrable, and for each $x \in X$, the set

$$
\left\{u \in \mathbb{P}: \varphi_{n}(x, u)=\mathbf{M}_{n}(x)\right\} \quad \text { and } \quad\left\{u \in \mathbb{P}: \varphi_{n}(x, u)=\mathbf{m}_{n}(x)\right\}
$$

are nonempty closed subsets.

(iii) $(n+k) \mathbf{M}_{n+k} \leq n \mathbf{M}_{n}+k D_{n} \mathbf{M}_{k}$ and $(n+k) \mathbf{m}_{n+k} \geq n \mathbf{m}_{n}+k D_{n} \mathbf{m}_{k}$ for $k, n \in \mathbb{N}$. 
Proof. Combining the definition of $\mathbf{M}_{n}$ and $\mathbf{m}_{n}$ in (29) and (16), we get that

$$
\begin{aligned}
& \mathbf{M}_{n}(x)=\sup _{u \in \mathbb{P}} \frac{1}{n} \int_{\Omega_{x}} \log \|\mathcal{A}(\omega, n) u\| d W_{x}(\omega), \\
& \mathbf{m}_{n}(x)=\inf _{u \in \mathbb{P}} \frac{1}{n} \int_{\Omega_{x}} \log \|\mathcal{A}(\omega, n) u\| d W_{x}(\omega) .
\end{aligned}
$$

Consequently, assertion (i) follows from Lemma 4.4 (ii).

By Lemma 4.4 (iii), $\varphi_{n}$ belongs to $L_{\mu}^{1}(\mathscr{C}(\mathbb{P}))$ and $\varphi_{n}$ is a Borel function. Fixing a sequence $\left(u_{j}\right)_{j=1}^{\infty} \subset \mathbb{P}$ which is dense in $\mathbb{P}$, and using the continuity of $\mathbb{P} \ni v \mapsto \varphi_{n}(x, v)$ for each $x \in X$, we see that $\mathbf{M}_{n}(x)=\sup _{j \geq 1} \varphi_{n}\left(x, u_{j}\right)$ for all $x \in X$. Hence, $\mathbf{M}_{n}$ is a Borel function. Similarly, we can show that $\mathbf{m}_{n}$ is also a Borel function. The $\mu$-integrability of $\mathbf{M}_{n}$ and $\mathbf{m}_{n}$ follows from combining assertion (i) and Lemma 4.4 (iii). We also infer from the continuity of $\mathbb{P} \ni v \mapsto \varphi_{n}(x, v)$ for each $x \in X$ that the two sets considered in (ii) are nonempty closed. This implies assertion (ii).

We only give the proof of the first inequality in assertion (iii) since the second one can be proved similarly. Fix $x_{0} \in X$ and $n, k \in \mathbb{N}$. So we have to show that

$$
(n+k) \mathbf{M}_{n+k}\left(x_{0}\right) \leq n \mathbf{M}_{n}\left(x_{0}\right)+k\left(D_{n} \mathbf{M}_{k}\right)\left(x_{0}\right) .
$$

Fix a universal covering $\phi: \mathbb{D} \rightarrow L_{x_{0}}$ with $\phi(0)=x_{0}$ as in (1). Let $f_{u}$ be the specialization of $\mathcal{A}$ at $\left(x_{0}, u\right)$. By assertion (ii) let $u_{0} \in \mathbb{P}$ be such that $\mathbf{M}_{n+k}\left(x_{0}\right)=\varphi_{n}\left(x_{0}, u_{0}\right)$. By Lemma 4.4 (ii) we have that

$$
p \varphi_{p}(x, u)=\mathbb{E}_{x}[\log \|\mathcal{A}(\bullet, p) u\|], \quad p \in \mathbb{N}, u \in \mathbb{P} .
$$

Putting this and (20) and (11) together, we may write

$(n+k) \mathbf{M}_{n+k}\left(x_{0}\right)=(n+k) \varphi_{n}\left(x_{0}, u_{0}\right)=\left(D_{n+k} f_{u_{0}}\right)(0)=\left(D_{n} f_{u_{0}}\right)(0)+\left(D_{n}\left(D_{k} f_{u_{0}}-f_{u_{0}}\right)\right)(0)$.

On the other hand, combining (29) and (22), we infer that

$$
p \mathbf{M}_{p}(x)=\max _{u \in \mathbb{P}}\left(D_{p} f_{u}\right)(\xi)-f_{u}(\xi), \quad p \in \mathbb{N}, x \in L_{x_{0}}, \xi \in \mathbb{D} \text { with } x=\phi(\xi) .
$$

Since $f_{u_{0}}(0)=0$ by (20), it follows that

$$
n \mathbf{M}_{n}\left(x_{0}\right) \geq\left(D_{n} f_{u_{0}}\right)(0),
$$

and that $k \mathbf{M}_{k}(\phi(\xi)) \geq\left(D_{k} f_{u_{0}}\right)(\xi)-f_{u_{0}}(\xi), \xi \in \mathbb{D}$. Applying (12) to the function $\mathbf{M}_{k}$ and to $\phi: \mathbb{D} \rightarrow L_{x_{0}}$, and using the latter inequality and the fact that $D_{n}$ is a positive operator yields that

$$
k\left(D_{n} \mathbf{M}_{k}\right)\left(x_{0}\right)=\left(D_{n}\left(k \mathbf{M}_{k}\right)\right)(\phi(0)) \geq\left(D_{n}\left(D_{k} f_{u_{0}}-f_{u_{0}}\right)\right)(0) .
$$

Inserting this and (34) into the last line of (33), we obtain (32). This completes the proof of (iii).

Lemma 4.8. The unique Lyapunov exponent $\chi$ of $\mathcal{A}$ satisfies

$$
\lim _{n \rightarrow \infty} \int_{X} \mathbf{M}_{n}(x) d \mu(x)=\chi=\lim _{n \rightarrow \infty} \int_{X} \mathbf{m}_{n}(x) d \mu(x) .
$$

Proof. We only prove the first equality since the proof of the second one is similar. Set $a_{n}:=n \int_{X} \mathbf{M}_{n}(x) d \mu(x)$ for $n \geq 1$. By Lemma 4.7 (iii), we get that $(n+k) \mathbf{M}_{n+k} \leq$ $n \mathbf{M}_{n}+k D_{n} \mathbf{M}_{k}$. Integrating both sides of this inequality and using that $\mu$ is $D_{n}$-invariant 
(see Definition 2.4), we obtain that $a_{n+k} \leq a_{n}+a_{k}$. So $\lim _{n \rightarrow \infty} \frac{a_{n}}{n}$ exists and is equal to $a:=\inf _{n \geq 1} \frac{a_{n}}{n}$. Set

$$
\Pi_{n}(x):=\left\{(x, u) \in X \times \mathbb{P}: \varphi_{n}(x, u)=\mathbf{M}_{n}(x)\right\}, \quad x \in X .
$$

Since we know from Lemma 4.7 (ii) that $\mathbf{M}_{n}$ is measurable and that $\Pi_{n}(x)$ is a closed set for each $x \in X$, we can choose by [9, Theorem III.6] a measurable map $u_{n}: X \rightarrow \mathbb{P}$ such that $\left(x, u_{n}(x)\right) \in \Pi_{n}$ for $\mu$-almost every $x \in X$. For $n \geq 1$ let $\nu_{n} \in L_{\mu}^{\infty}(\mathscr{M}(\mathbb{P}))$ be defined as follows: for each $x \in X, \nu_{n}(x)$ is the Dirac mass at $u_{n}(x)$. Next, applying Lemma 4.5 to the sequence $\left(\nu_{n}\right)_{n=1}^{\infty}$ yields a subsequence $\left(\nu_{n_{j}}\right)_{j=1}^{\infty}$ such that $\frac{1}{n_{j}} \sum_{k=0}^{n_{j}-1} D_{k} \nu_{n_{j}}$ converges weakly to a probability measure $\nu \in \operatorname{Har}_{\mu}(X \times \mathbb{P})$. Moreover, by Lemma 4.5 (ii) we have that

$$
\lim _{j \rightarrow \infty} \int_{X} \varphi_{n_{j}}\left(x, u_{n_{j}}(x)\right) d \mu(x)=\int_{X \times \mathbb{P}} \varphi d \nu .
$$

By Theorem 4.6, the right hand side is equal to $\chi$. A combination of Lemma 4.4 (ii) and (29) shows that the left hand side is equal to $\lim _{j \rightarrow \infty} a_{n_{j}}$ which is $a$. Hence, we have shown that $a=\chi$, which amounts to $\lim _{n \rightarrow \infty} \int_{X} \mathbf{M}_{n}(x) d \mu(x)=\chi$, as desired.

Now we discuss some properties of the operator $D=D_{1}$ given in (10). Since $p(x, y, t) \geq$ 0 (see [10]) and $\mu$ is $D$-invariant (see Definition 2.4), we infer that $D$ is a positive linear operator acting on the space $L^{1}(X, \mu)$ and that

$$
\|D f\|_{L^{1}(X, \mu)} \leq\|f\|_{L^{1}(X, \mu)} .
$$

In other words, $D$ is Markovian in the sense of Akcoglu-Sucheston [1]. Moreover,

$$
\|D f\|_{L^{\infty}(X, \mu)} \leq\|f\|_{L^{\infty}(X, \mu)} .
$$

On the other hand, since $T$ is extremal, Theorem 2.6 (iii) says that $\mu$ is ergodic. Consequently, applying [8, Theorem 2.5.5] (see also [17]) yields the following

Theorem 4.9. For every $f \in L^{1}(X, \mu), \frac{1}{n} \sum_{i=0}^{n-1} D_{i} f$ tends to $\int_{X} f d \mu$ as $n \rightarrow \infty \mu$-almost everywhere.

A sequence $\left(f_{n}\right)_{n=0}^{\infty} \subset L^{1}(X, \mu)$ is said to be subadditive if $f_{n+k} \leq f_{n}+D_{n} f_{k}$ for all $n, k \in \mathbb{N}$. Using Theorem 4.9 and the fact that $D$ is Markovian, we may restate AkcogluSucheston ratio ergodic theorem for subadditive sequences as follows.

Theorem 4.10. (Akcoglu-Sucheston [1]) Let $\left(f_{n}\right)_{n=0}^{\infty} \subset L^{1}(X, \mu)$ be a subadditive sequence such that $\gamma:=\inf _{n \geq 1} \int_{X} \frac{f_{n}}{n} d \mu>-\infty$. Then $\lim _{n \rightarrow \infty} \frac{f_{n}}{n}=\gamma \mu$-almost everywhere.

Now we arrive at the

End of the proof of Theorem 4.1. By Lemma 4.7 (ii)-(iii) $\left(n \mathbf{M}_{n}\right)_{n=1}^{\infty}$ and $\left(-n \mathbf{m}_{n}\right)_{n=1}^{\infty}$ are subadditive sequences. By Lemma 4.8 we have that

$$
\lim _{n \rightarrow \infty} \int_{X} \mathbf{M}_{n}(x) d \mu(x)=\chi=\lim _{n \rightarrow \infty} \int_{X} \mathbf{m}_{n}(x) d \mu(x) .
$$

Consequently, applying Theorem 4.10 to $\left(n \mathbf{M}_{n}\right)_{n=1}^{\infty}$ and $\left(-n \mathbf{m}_{n}\right)_{n=1}^{\infty}$ yields the existence of a Borel set $Y_{0} \subset Y$ of full $\mu$-measure such that

$$
\lim _{n \rightarrow \infty} \mathbf{M}_{n}(x)=\chi=\lim _{n \rightarrow \infty} \mathbf{m}_{n}(x)
$$

for every $x \in Y_{0}$. This completes the proof in the case of a single Lyapunov exponent, and hence the general case follows from the reduction made in Subsection 4.1. 


\section{Proof of THE SECOND PART OF THE MAIN THEOREM}

We begin this section with some preparatory results on the heat diffusions on the Poincaré disc $\left(\mathbb{D}, g_{P}\right)$. In what follows, for $a \in \mathbb{D}$ and $R>0$, we denote by $\mathbb{D}(a, R)$ the Poincaré ball $\left\{\xi \in \mathbb{D}: \operatorname{dist}_{P}(a, \xi)<R\right\}$. For every $R \in \mathbb{R}$, let $[R]$ be the integer part of $R$, i.e., $[R]=n$ if and only if $n \in \mathbb{Z}$ and $n \leq R<n+1$.

Lemma 5.1. Let $f \in \mathscr{C}^{2}(\mathbb{D})$ be such that $f,|d f|_{P}$ and $\Delta f$ are moderate functions on $\mathbb{D}$. Then

$$
\left(D_{t} f\right)(\xi)-f(\xi)=\int_{0}^{t}\left(D_{s} \Delta f\right)(\xi) d s, \quad t \in \mathbb{R}^{+}, \xi \in \mathbb{D} .
$$

Proof. We follow along the same lines as the proofs of Candel in [7, Proposition 8.11]. Indeed, recall from [7] the following Dynkin's formula (see [18] or [8, Theorem C.8.1] for a proof): for every function $f$ in the space $\mathscr{C}_{0}^{2}(\mathbb{D})$ of $\mathscr{C}^{2}$-differentiable functions on $\mathbb{D}$ with compact support, it holds that

$$
\mathbb{E}_{\xi}\left[f \circ \pi_{t}\right]-f(\xi)=\mathbb{E}_{\xi}\left[\int_{0}^{t}(\Delta f) \circ \pi_{s} d s\right], \quad t \in \mathbb{R}^{+}, \xi \in \mathbb{D},
$$

where the projection $\pi_{t}: \Omega \rightarrow X$ is given by $\pi_{t}(\omega):=\omega(t), \omega \in \Omega, t \in \mathbb{R}^{+}$. Using identity (17), the above formula can be rewritten, less stochastically and more analytically, as follows:

$$
\left(D_{t} f\right)(\xi)-f(\xi)=\int_{0}^{t}\left(D_{s} \Delta f\right)(\xi) d s, \quad t \in \mathbb{R}^{+}, \xi \in \mathbb{D},
$$

where $f \in \mathscr{C}_{0}^{2}(\mathbb{D})$. So it remains to extend (35) to the case where $f$ only satisfies the growth assumption of the lemma.

Let $\left(\xi_{k}\right)_{k=0}^{\infty}$ be a sequence of points in $\mathbb{D}$ constructed as follows. Set $\xi_{0}:=0$ and $p_{0}:=0$. For every $n \geq 1$, suppose that we have already defined $\xi_{j}$ with $j \leq p_{n-1}$, we want to construct an integer $p_{n}>p_{n-1}$ and the new points $\xi_{j}$ with $p_{n-1}<j \leq p_{n}$ as follows. Let $p_{n}=1+\left[2 \pi e^{n}\right]+p_{n-1}$. Let $\xi_{p_{n}}$ be the unique common point lying on both the positive real axis of $\mathbb{C}$ and the circle $\partial \mathbb{D}(0, n)$. Consider the $\left(1+\left[2 \pi e^{n}\right]\right)$-sided regular polygon inscribed in the circle $\partial \mathbb{D}(0, n)$ having $\xi_{p_{n}}$ as a vertex. Let $\xi_{p_{n}-1}, \ldots, \xi_{p_{n-1}+1}$ be the remaining vertices of this polygon. So the Poincaré distance between two consecutive vertices of the polygon is $\leq 1$ since the Poincaré length of $\partial \mathbb{D}(0, n)$ is $2 \pi e^{n}$. Continuing this process, we obtain a sequence $\left(\xi_{k}\right)_{k=0}^{\infty} \subset \mathbb{D}$. Note that $\operatorname{dist}_{P}\left(\xi_{p_{n}}, \xi_{p_{n+1}}\right)=1$ for all $n \in \mathbb{N}$.

From this construction we make the following observations:

- the family of balls $\left(\mathbb{D}\left(\xi_{k}, 4\right)\right)_{k=0}^{\infty}$ is an open cover of $\mathbb{D}$;

- there is an integer $N>1$ such that for every $a \in \mathbb{D}$, the cardinal of the set $\left\{k \in \mathbb{N}: a \in \mathbb{D}\left(\xi_{k}, 8\right)\right\}$ is $\leq N$.

In particular, the family of balls $\left(U_{k}:=\mathbb{D}\left(\xi_{k}, 8\right)\right)_{k=0}^{\infty}$ is locally finite in $\mathbb{D}$. Fix a smooth compactly supported function $\psi: \mathbb{D}(0,8) \rightarrow[0,1]$ such that $\psi=1$ on $\mathbb{D}(0,4)$. For $k \in \mathbb{N}$ fix an automorphism $\tau_{k}$ of $\mathbb{D}$ which sends $\xi_{k}$ to 0 . Consider the sequence of functions $\left(\psi_{k}\right)_{k=1}^{\infty}$ defined by

$$
\psi_{k}:=\frac{\psi \circ \tau_{k}}{\sum_{j=0}^{\infty} \psi \circ \tau_{j}} \quad \text { on } U_{k}
$$


Using the above observations, we see easily that $\psi_{k}$ is a well-defined function in $\mathscr{C}_{0}^{\infty}\left(U_{k},[0,1]\right)$ for each $k$, and $\left(\psi_{k}\right)_{k=1}^{\infty}$ is a partition of unity subordinate to the cover $\left(U_{k}\right)_{k=0}^{\infty}$. Moreover, there is a global bound $c_{1}>0$ such that for all $k \in \mathbb{N}$,

$$
\left|d \psi_{k}\right|_{P} \leq c_{1} \text { and }\left|\Delta \psi_{k}\right| \leq c_{1} .
$$

For each $k \in \mathbb{N}$, let $f_{k}$ be the function in $\mathscr{C}_{0}^{2}(\mathbb{D})$ defined by

$$
f_{k}:=\sum_{j=1}^{k} \psi_{i} f
$$

By (35), we have that

$$
\left(D_{t} f_{k}\right)(\xi)-f_{k}(\xi)=\int_{0}^{t}\left(D_{s} \Delta f_{k}\right)(\xi) d s, \quad t \in \mathbb{R}^{+}, \xi \in \mathbb{D} .
$$

On the one hand, $f_{k} \rightarrow f$ uniformly on compact subsets of $\mathbb{D}$ as $k \rightarrow \infty$ and $\left|f_{k}\right| \leq|f|$. Therefore, we can show that the left hand side of (38) tends to $\left(D_{t} f\right)(\xi)-f(\xi)$ uniformly on compact subsets as $k \rightarrow \infty$.

We now examine the right hand side of (38). Using a holomorphic automorphism of $\mathbb{D}$ sending $\xi$ to 0 , we may suppose without loss of generality that $\xi=0$. Recall the sample-path space $\Omega_{0}$ and the Wiener measure $W_{0}$ from Subsection 2.3 . As $k \rightarrow \infty$, the functions $\Delta f_{k}$ converge to $\Delta f$ uniformly on compact sets, hence

$$
\int_{0}^{t} \Delta f_{k}(\omega(s)) d s \rightarrow \int_{0}^{t} \Delta f(\omega(s)) d s
$$

for each path $\omega \in \Omega_{0}$, since $\omega[0, t]$ is compact. Thus, $\int_{0}^{t} \Delta f_{k}(\bullet(s)) d s$ converge pointwise to $\int_{0}^{t} \Delta f(\bullet(s)) d s$ in $\Omega_{0}$. Each of the functions

$$
\Omega_{0} \ni \omega \mapsto \int_{0}^{t} \Delta f_{k}(\omega(s)) d s
$$

is integrable with respect to $W_{0}$. Now we show that the convergence is also dominated. Indeed, we infer easily from (36) and (37) that

$$
\left|\Delta f_{k}\right| \leq c_{2}|f|+|\Delta f|+c_{2}|d f|_{P} \quad \text { for some } c_{2}>0 \text { and for all } k \geq 1 \text {. }
$$

This implies that for every $\omega \in \Omega_{0}$,

$$
\begin{aligned}
\left|\int_{0}^{t} \Delta f_{k}(\omega(s)) d s\right| & \leq \int_{0}^{t}\left|\Delta f_{k}\right|(\omega(s)) d s \\
& \leq c_{2} \int_{0}^{t}|f|(\omega(s)) d s+\int_{0}^{t}|\Delta f|(\omega(s)) d s+c_{2} \int_{0}^{t}|d f|_{P}(\omega(s)) d s .
\end{aligned}
$$

Using the moderateness of $f, \Delta f$ and $|d f|_{P}$, we will show that each term on the righthand side of (39) is integrable with respect to $W_{0}$. Indeed, the moderateness of $f$ says that the first term is bounded from above by

$$
\int_{0}^{t} \exp \left(c^{\prime}+c^{\prime} \operatorname{dist}_{P}(\omega(t), 0)\right) d s \quad \text { for some } c^{\prime}>0 \text { depending only on } f \text {. }
$$


It has been shown in Remark 3.2 that

$$
\int_{\Omega_{0}} \exp \left(c^{\prime}+c^{\prime} \operatorname{dist}_{P}(\omega(t), 0)\right) d W_{0}(\omega)<\infty
$$

hence, by Fubini's theorem, the function

$$
\Omega_{0} \ni \omega \mapsto \int_{0}^{t}|f|(\omega(s)) d s
$$

is integrable with respect to $W_{0}$. Similarly, we can show that the remaining two functions (of $\omega$ ) on the right-hand side of (39) is integrable with respect to $W_{0}$. Consequently, by Lebesgue's dominated convergence,

$$
\mathbb{E}_{0}\left[\int_{0}^{t} \Delta f_{k}(\bullet(s)) d s\right] \rightarrow \mathbb{E}_{0}\left[\int_{0}^{t} \Delta f(\bullet(s)) d s\right] \quad \text { as } \quad k \rightarrow \infty .
$$

Putting this together with (16) and (17), it follows that $\int_{0}^{t}\left(D_{s} \Delta f_{k}\right)(0) d s$ converge to $\int_{0}^{t}\left(D_{s} \Delta f\right)(0) d s$ as $k \rightarrow \infty$. Thus, the right-hand side of (38) with $\xi=0$ converges to $\int_{0}^{t}\left(D_{s} \Delta f\right)(0) d s$ as $k \rightarrow \infty$. This, combined with the convergence of its left-hand side which has been previously shown, completes the proof of the lemma for $\xi=0$, and hence for every $\xi \in \mathbb{D}$.

Lemma 5.2. Let $\mathcal{A}$ be a strongly moderate cocycle. Then there is a constant $c>0$ such that for every $(x, u) \in X \times \mathbb{P}\left(\mathbb{K}^{d}\right)$, the specialization $f:=f_{x, u}$ satisfies the following two conditions:

- both $f$ and $|d f|_{P}$ are moderate functions with constant $c$;

- $|\Delta f| \leq c$ on $\mathbb{D}$.

Moreover, $\mathcal{A}$ is uniformly Lipschitz.

Proof. By Definition 2.8, there is a constant $c_{1}>0$ such that

$f$ is a moderate function with constant $c_{1}$ and $|\Delta f| \leq c_{1}$ on $\mathbb{D}$.

To complete the proof of the lemma, it suffices to show that $|d f|_{P} \leq c$ for some constant $c>c_{1}$ large enough. By Item 3. in Remark 2.9, it is sufficient to show that $|d f(0)|_{P} \leq c$.

Fix an arbitrary $0<r<1$. By Riesz representation formula for the disc $\{z \in \mathbb{C}:|z|<$ $r\}$ gives for $|z|<r$,

$$
f(z)=\frac{1}{2 \pi} \int_{\zeta \in \mathbb{C}:|\zeta|<r} \log \frac{r|z-\zeta|}{\left|r^{2}-z \bar{\zeta}\right|}(\Delta f) g_{P}+\frac{1}{2 \pi} \int_{0}^{2 \pi} \frac{1-|z / r|^{2}}{\left|e^{i \theta}-z / r\right|^{2}} f\left(r e^{i \theta}\right) d \theta .
$$

We deduce from (40) that there is a constant $c_{2}>c_{1}$ depending only on $c_{1}$ and $r$ such that $|f(z)|<c_{2}$ and that $|\Delta f(z)|<c_{2}$ for all $|z|<r$. Using this and performing the derivative of the right hand side of (41) with respect to $z$, we obtain that $|d f(0)|_{P} \leq c$ for some constant $c>c_{2}$ depending only on $c_{2}$ and $r$.

Lemma 5.3. Let $f \in \mathscr{C}^{2}(\mathbb{D})$ be such that both $f$ and $|d f|_{P}$ are moderate functions on $\mathbb{D}$ and that $\Delta f$ is bounded on $\mathbb{D}$. Then for every $R>1$,

$$
\int_{0}^{1} f\left(r_{R} e^{2 \pi i \theta}\right) d \theta=\left(D_{[R]} f\right)(0)+O\left(R^{1 / 2} \sqrt{\log R}\right)
$$


where $r_{R}$ is calculated according to the conversion rule (5) and $[R]$ is the integer part of $R$. Proof. By Riesz representation formula we have that

$$
\int_{0}^{1} f\left(r e^{2 \pi i \theta}\right) d \theta-f(0)=\frac{1}{2 \pi} \int \log ^{+} \frac{r}{|\zeta|}(\Delta f) g_{P}
$$

where $\log ^{+}:=\max \{\log , 0\}$. For $R>0$ let

$$
M_{R}:=\int_{\mathbb{D}} \log ^{+} \frac{r}{|\zeta|} g_{P}=\int_{\mathbb{D}} \log ^{+} \frac{r}{|\zeta|} \frac{2}{\left(1-|\zeta|^{2}\right)^{2}} i d \zeta \wedge d \bar{\zeta}
$$

Recall from the proof of [14, Lemma 7.6] that there is a constant $c>0$ such that the following estimate holds

$$
\left|\frac{1}{M_{R}} \int \log ^{+} \frac{r}{|\zeta|} u g_{P}-\frac{2 \pi}{M_{R}} \int_{0}^{\frac{M_{R}}{2 \pi}}\left(D_{t} u\right)(0) d t\right| \leq c R^{-1 / 2} \sqrt{\log R}\|u\|_{\infty}
$$

for all $R \in \mathbb{R}^{+}$and all bounded measurable functions $u$ on $\mathbb{D}$. Since $\Delta f$ is bounded, the above inequality, applied to $\Delta f$, gives that

$$
\left|\frac{1}{M_{R}} \int \log ^{+} \frac{r}{|\zeta|}(\Delta f) g_{P}-\frac{2 \pi}{M_{R}} \int_{0}^{\frac{M_{R}}{2 \pi}}\left(D_{t} \Delta f\right)(0) d t\right| \leq c R^{-1 / 2} \sqrt{\log R}\|\Delta f\|_{\infty}
$$

Inserting (42) into the first term of the left hand side, we get that

$$
\left|\frac{2 \pi}{M_{R}}\left(\int_{0}^{1} f\left(r e^{2 \pi i \theta}\right) d \theta-f(0)\right)-\frac{2 \pi}{M_{R}} \int_{0}^{\frac{M_{R}}{2 \pi}}\left(D_{t} \Delta f\right)(0) d t\right| \leq c R^{-1 / 2} \sqrt{\log R}\|\Delta f\|_{\infty} .
$$

Moreover, a direct computation shows that $\left|M_{R}-2 \pi R\right|$ is bounded by a constant and it is clear that $|R-[R]|<1$. Putting this together with the estimate $\left\|D_{t} \Delta f\right\|_{\infty} \leq\|\Delta f\|_{\infty}<\infty$ for all $t \in \mathbb{R}^{+}$, we infer from the last line that

$$
\left|\frac{1}{R}\left(\int_{0}^{1} f\left(r e^{2 \pi i \theta}\right) d \theta-f(0)\right)-\frac{1}{[R]} \int_{0}^{[R]}\left(D_{t} \Delta f\right)(0) d t\right| \leq c R^{-1 / 2} \sqrt{\log R}\|\Delta f\|_{\infty} .
$$

Applying Lemma 5.1 to the second term on the left hand side yields that

$$
\left|\frac{1}{R}\left(\int_{0}^{1} f\left(r e^{2 \pi i \theta}\right) d \theta-f(0)\right)-\frac{1}{[R]}\left(\left(D_{[R]} f\right)(0)-f(0)\right)\right| \leq c R^{-1 / 2} \sqrt{\log R}\|\Delta f\|_{\infty} .
$$

The proof is thereby completed.

Now we are in the position to complete the proof of the Main Theorem.

End of the proof of assertion (ii) of Theorem 1.4. The proof is divided into two steps. Let $Y_{0}$ be the Borel set of full $\mu$-measure given by Theorem 4.1,

Step 1: Identity (9) (namely, $\lim _{R \rightarrow \infty} \mathscr{E}\left(x, H_{i}(x), R\right)=\chi_{i}$ ) holds for each $x \in Y_{0}$ and for each $1 \leq i \leq m$.

Fix an index $1 \leq i_{0} \leq m$ and a point $x_{0} \in Y_{0}$. Let $\phi_{x_{0}}: \mathbb{D} \rightarrow L=L_{x_{0}}$ be the universal covering map given in (11). For each vector $v \in H_{i_{0}}\left(x_{0}\right) \backslash\{0\}$, let $f_{v}$ be the specialization of $\mathcal{A}$ at $\left(x_{0},[v]\right)$ (see formula (19)). By (20), we have that

$$
\mathbb{E}_{x_{0}}\left[\log \frac{\|\mathcal{A}(\bullet, R) v\|}{\|v\|}\right]=\left(D_{R} f_{v}\right)(0), \quad R>0 .
$$


On the other hand, since $x_{0} \in Y_{0}$, Theorem 4.1 tells us that

$$
\lim _{n \rightarrow \infty} \frac{1}{n} \inf _{v \in H_{i_{0}}\left(x_{0}\right) \backslash\{0\}} \mathbb{E}_{x_{0}}\left[\log \frac{\|\mathcal{A}(\bullet, n) v\|}{\|v\|}\right]=\lim _{n \rightarrow \infty} \frac{1}{n} \sup _{v \in H_{i_{0}}\left(x_{0}\right) \backslash\{0\}} \mathbb{E}_{x_{0}}\left[\log \frac{\|\mathcal{A}(\bullet, n) v\|}{\|v\|}\right]=\chi_{i_{0}} .
$$

Therefore, we deduce from the last two lines that

$$
\lim _{R \rightarrow \infty} \inf _{v \in H_{i_{0}}\left(x_{0}\right) \backslash\{0\}} \frac{1}{R}\left(D_{[R]} f_{v}\right)(0)=\chi_{i_{0}}=\lim _{R \rightarrow \infty} \sup _{v \in H_{i_{0}}\left(x_{0}\right) \backslash\{0\}} \frac{1}{R}\left(D_{[R]} f_{v}\right)(0) .
$$

Since $\mathcal{A}$ is strongly moderate, Lemma 5.2 says that $f_{v}$ satisfies the assumption of Lemma 5.3. Consequently, using this lemma the last estimate implies that

$$
\inf _{v \in H_{i_{0}}\left(x_{0}\right) \backslash\{0\}} \frac{1}{R} \int_{0}^{1} f_{v}\left(r_{R} e^{2 \pi i \theta}\right) d \theta=\chi_{i_{0}}=\sup _{v \in H_{i_{0}}\left(x_{0}\right) \backslash\{0\}} \frac{1}{R} \int_{0}^{1} f_{v}\left(r_{R} e^{2 \pi i \theta}\right) d \theta .
$$

Hence, $\mathscr{E}\left(x_{0}, H_{i_{0}}\left(x_{0}\right), R\right) \rightarrow \chi_{i_{0}}$ as $R \rightarrow \infty$. Step 1 is thereby completed.

Step 2: There exists a leafwise saturated Borel set $Y \subset X$ of full $\mu$-measure such that $\lim _{R \rightarrow \infty} \mathscr{E}\left(x, H_{i}(x), R\right)=\chi_{i}$ for each $x \in Y$ and each $1 \leq i \leq m$.

Let $Y$ be the saturation of $Y_{0}$, that is, $Y:=\bigcup_{x \in Y_{0}} L_{z}$. Since $Y_{0}$ is of full $\mu$-measure, so is $Y$. By shrinking $Y$ a little, we may assume that $Y$ is leafwise saturated Borel set of full $\mu$-measure. Fix an index $i_{0}$ and a point $x_{1} \in Y$. We need to show that $\lim _{R \rightarrow \infty} \mathscr{E}\left(x_{1}, H_{i_{0}}\left(x_{1}\right), R\right)=\chi_{i_{0}}$. Let $\phi_{x_{1}}: \mathbb{D} \rightarrow L=L_{x_{1}}$ be the universal covering map given in (1). Pick a point $x_{2} \in L \cap Y_{0}$. Pick $\xi_{2} \in \phi_{x_{1}}^{-1}\left(x_{2}\right)$. Fix a path $\omega \in \Omega_{0}$ such that $\omega(1)=\xi_{2}$. For each $v \in H_{i_{0}}\left(x_{1}\right)$, we set $u_{v}:=\mathcal{A}\left(\phi_{x_{1}} \circ \omega, 1\right) v \in H_{i_{0}}\left(x_{2}\right)$, and let $f_{1, v}$ (resp. $\left.f_{2, v}\right)$ be the specialization of $\mathcal{A}$ at $\left(x_{1},[v]\right)$ (resp. at $\left(x_{2},\left[u_{v}\right]\right)$ ).

Since $x_{2} \in Y_{0}$ and $H_{i_{0}}\left(x_{1}\right) \ni v \mapsto u_{v} \in H_{i_{0}}\left(x_{2}\right)$ is an isomorphism, we infer from identity (43) applied to $x_{2}$ that

$$
\lim _{R \rightarrow \infty} \inf _{v \in H_{i_{0}}\left(x_{1}\right) \backslash\{0\}} \frac{1}{R}\left(D_{[R]} f_{2, v}\right)(0)=\chi_{i_{0}}=\lim _{R \rightarrow \infty} \sup _{v \in H_{i_{0}}\left(x_{1}\right) \backslash\{0\}} \frac{1}{R}\left(D_{[R]} f_{2, v}\right)(0) .
$$

Recall from identity (21) and the expression of $u_{v}$ in terms of $v$ that

$$
f_{2, v}(\xi)=f_{1, v}(\xi)-f_{1, v}\left(\xi_{2}\right), \quad \xi \in \mathbb{D}, v \in H_{i_{0}}\left(x_{1}\right) .
$$

Inserting this into (44), we get that

$$
\lim _{R \rightarrow \infty} \inf _{v \in H_{i_{0}}\left(x_{1}\right) \backslash\{0\}} \frac{1}{R}\left(D_{[R]} f_{1, v}\right)(0)=\chi_{i_{0}}=\lim _{R \rightarrow \infty} \sup _{v \in H_{i_{0}}\left(x_{1}\right) \backslash\{0\}} \frac{1}{R}\left(D_{[R]} f_{1, v}\right)(0) .
$$

Using this we argue as we did from (43) to the end of the proof of Step 1. Consequently, we conclude that $\mathscr{E}\left(x_{0}, H_{i_{0}}\left(x_{1}\right), R\right) \rightarrow \chi_{i_{0}}$ as $R \rightarrow \infty$. So the last step and hence the proof of the Main Theorem is complete.

\section{APPLICATIONS AND CONCLUDING REMARKS}

Let $S$ be a compact Riemann surface of genus $>1, d \geq 1$ an integer, and $\mathbb{K} \in\{\mathbb{R}, \mathbb{C}\}$. Let $\rho: \pi_{1}(S) \rightarrow \mathrm{GL}(d, \mathbb{K})$ be a representation; this is the same as a local system $H \rightarrow S$ over $S$ with fiber $\mathbb{K}^{d}$. In fact, it is well-known that a local system is equivalent to a vector bundle endowed with a flat connection. For $x \in S$ denote by $H_{x}$ the fiber at $x$ of the local system. Consider $S$ as a lamination consisting of a single leaf and let $\Omega(S)$ be the sample-path space associated to $S$. 
For every $\omega \in \Omega(S)$ and $t \in \mathbb{R}^{+}$and $v \in H_{\omega(0)}$, let $\operatorname{hol}_{\omega, t} v$ be the the image of $v$ in $H_{\omega(t)}$ by the holonomy map via parallel transport (with respect to the Gauss-Manin connection) along the path $\omega[0, t]$.

We equip the vector bundle $H \rightarrow S$ with a Riemannian (resp. Hermitian) metric $h$. An identifier $\tau$ of $H \rightarrow S$ is a smooth map which associates to each point $x \in X$ a linear isometry $\tau(x): H_{x} \rightarrow \mathbb{K}^{d}$, that is, a $\mathbb{K}$-linear morphism such that

$$
\|\tau(x) v\|=\|v\|_{h}, \quad v \in H_{x}, x \in S,
$$

where the norm in the left hand side is the Euclidean norm (see [26, Section 3.1]). The existence of such a map $\tau$ can be proved using a partition of unity on $S$.

Consider the map $\mathcal{A}: \Omega(S) \times \mathbb{R}^{+} \rightarrow \mathrm{GL}(d, \mathbb{K})$ defined as follows.

$$
\mathcal{A}(\omega, t):=\tau(\omega(t)) \circ\left(\operatorname{hol}_{\omega, t}\right)(\omega(0)) \circ \tau^{-1}(\omega(0)), \quad \omega \in \Omega, t \in \mathbb{R}^{+} .
$$

It can be checked that $\mathcal{A}$ is a cocycle in the sense of Definition 1.1. We say that $\mathcal{A}$ is the associated cocycle of the representation $\rho$ and the identifier $\tau$. Since $\mathcal{A}$ is clearly $\mathscr{C}^{2}$-differentiable, we infer from Proposition 2.10 that it is strongly moderate.

On the other hand, we deduce from the assumption on $S$ that the Poincaré metric $g_{P}$ on $S$ is a nonzero finite measure. So in formula (2) we choose $T:=1$ and hence $\mu=g_{P}$ is an ergodic harmonic measure.

Therefore, we are in the position to apply Corollary 1.5 to $\mathcal{A}$. Consequently, we obtain the following result which characterizes the Lyapunov exponents of $\mathcal{A}$ both dynamically and geometrically.

Proposition 6.1. Let $\rho: \pi_{1}(S) \rightarrow \mathrm{GL}(d, \mathbb{K})$ be a representation as above and $\mathcal{A}$ its associated cocycle. Then there exist a number $m \in \mathbb{N}$ together with $m$ integers $d_{1}, \ldots, d_{m} \in \mathbb{N}$ such that the following properties hold:

(i) For each $x \in S$ there exists a decomposition of $\mathbb{K}^{d}$ as a direct sum of $\mathbb{K}$-linear subspaces

$$
\mathbb{K}^{d}=\oplus_{i=1}^{m} H_{i}(x),
$$

such that $\operatorname{dim} H_{i}(x)=d_{i}$ and $\mathcal{A}(\omega, t) H_{i}(x)=H_{i}(\omega(t))$ for all $\omega \in \Omega_{x}$ and $t \in \mathbb{R}^{+}$. For each $1 \leq i \leq m$ and each $x \in S$, let $V_{i}(x):=\oplus_{j=i}^{m} H_{j}(x)$. Set $V_{m+1}(x) \equiv\{0\}$.

(ii) There are real numbers

$$
\chi_{m}<\chi_{m-1}<\cdots<\chi_{2}<\chi_{1}
$$

such that for each $x \in S$, there is a set $F_{x} \subset \Omega_{x}$ of full $W_{x}$-measure such that for every $1 \leq i \leq m$ and every $v \in V_{i}(x) \backslash V_{i+1}(x)$ and every $\omega \in F_{x}$,

$$
\lim _{t \rightarrow \infty, t \in \mathbb{R}^{+}} \frac{1}{t} \log \frac{\|\mathcal{A}(\omega, t) v\|}{\|v\|}=\chi_{i} .
$$

Moreover, for every $x \in S$ and for every $\omega \in F_{x}$,

$$
\lim _{t \rightarrow \infty, t \in \mathbb{R}^{+}} \frac{1}{t} \log \|\mathcal{A}(\omega, t)\|=\chi_{1}
$$

(iii) For each $x \in S$, there is a set $G_{x} \subset[0,1)$ of full Lebesgue measure such that equalities (7)-(8) hold for all $\theta \in G_{x}$.

(iv) For each $x \in S$, equality (9) holds.

Here $\|\cdot\|$ denotes the standard Euclidean norm of $\mathbb{K}^{d}$. 
The following decomposition at each fiber of the local system $H \rightarrow S$

$$
H_{x}:=\oplus_{i=1}^{m} H_{i, x},
$$

where $H_{i, x}:=\tau(x)^{-1} H_{i}(x), x \in S$, is called the Oseledec decomposition at $x$ of the representation $\rho$. The set of numbers $\chi_{m}<\chi_{m-1}<\cdots<\chi_{2}<\chi_{1}$ is called the Lyapunov spectrum of $\rho$. The decreasing sequence of subspaces of $H_{x}$ given by:

$$
\{0\} \equiv V_{m+1, x} \subset V_{m, x} \subset \cdots \subset V_{1, x}=H_{x},
$$

where $V_{i, x}:=\tau(x)^{-1} V_{i}(x), x \in S$, is called the Lyapunov filtration at $x$ of $\rho$. Notice that the compactness of $S$ and the requirement (46) imply that the Lyapunov spectrum, the Oseledec decompositions as well as the Lyapunov filtrations of $\rho$ are, in fact, independent of the choice of any metric $h$ as well as any identifier $\tau$.

Now we discuss another approach to define Lyapunov exponents of a linear representation which has been used by Bonatti, Gómez-Mont and many others (see [4] and the references therein). This approach relies on the geodesic flows.

Let $\rho: \pi_{1}(S) \rightarrow \mathrm{GL}(d, \mathbb{K})$ be a representation as above, and $H \rightarrow S$ its associated local system. Let $T^{1} S$ be the unit tangent bundle of $S$ and $\pi: T^{1} S \rightarrow S$ the natural projection. Each $y \in T^{1} S$ corresponds, in a natural way, to a unique unit-speed geodesic ray $\gamma_{x, \theta}$, where $x:=\pi(y)$ and $\theta$ is the direction of $y$ at $x$. Endow $T^{1} S$ with the Liouville measure $\mu$. Under the identification $y \equiv(x, \theta), \mu$ may be written as the product of the measure $g_{P}$ for $x \in S$ and the Lebesgue measure for $\theta \in[0,1)$. This is the product structure of the Liouville measure. Moreover, $\mu$ is an invariant measure which is ergodic with respect to the geodesic flow $\left(g_{t}\right)_{t \in \mathbb{R}^{+}}$on $S$. Using $\pi$, we may view $H$ as a local system over $T^{1} S$ whose fiber at $y \in T^{1} S$ is set to be simply the fiber $H_{\pi(y)}$. We make the following observation: For each $y \in T^{1} S, g_{t}(y): H_{y} \rightarrow H_{g_{t} y}$ is an invertible linear map between fibers. Fix an identifier $\tau$ and a metric $h$ of $H \rightarrow S$ as above and let $\mathcal{A}$ be the associated cocycle of the representation $\rho$ and the identifier $\tau$. Identifying the fibers of $H \rightarrow T^{1} S \stackrel{\pi}{\rightarrow} S$ with $\mathbb{K}^{d}$ using $\tau$, we get that

$$
\mathcal{A}\left(\gamma_{x, \theta}, t\right)=g_{t}(y)
$$

for every $t \in \mathbb{R}^{+}$and every unit-speed geodesic ray $\gamma_{x, \theta}$ that represents $y \in T^{1} S$. Let $\left\|g_{t}(y)\right\|$ be the norm of the linear map $g_{t}(y)$.

Since $S$ is compact we see easily that

$$
\int_{T^{1} S} \sup _{t \in[-1,1]}\left\|g_{t}(y)\right\| d \mu(y)<\infty .
$$

By the Oseledec multiplicative ergodic theorem (see [23, 29]), there exist numbers $\lambda_{1}>$ $\lambda_{2}>\cdots>\lambda_{r}$, called Lyapunov exponents, and a measurable $g_{t}$-invariant decomposition of the bundle

$$
H_{y}=\bigoplus_{i=1}^{r} H_{y}^{\lambda_{i}}
$$

such that for $\mu$-almost every $y \in T^{1} S$ and for every $v \in H_{y}^{\lambda_{i}}$, we have the asymptotic growth of norm

$$
\lim _{t \rightarrow \pm \infty} \frac{1}{t} \log \left\|g_{t} v\right\|=\lambda_{i} .
$$


This, combined with (47) and Proposition 6.1 (iii) and the product structure of the Liouville measure, implies that $\left\{\lambda_{1}, \ldots, \lambda_{r}\right\} \equiv\left\{\chi_{1}, \ldots, \chi_{m}\right\}$ and the two Oseledec decompositions (namely, Proposition 6.1 (i) and (48)) are the same. Consequently, we infer the following remarkable property. The subspaces $H_{y}^{\lambda_{i}}$ in the decomposition (48) depend only on $x:=\pi(y)$; in particular, they are independent of the direction $\theta$ while identifying $y$ with $(x, \theta)$.

In summary, in this particular example, our approach and the other one using the geodesic flows give the same Oseledec decomposition. However, our approach yields a stronger result. Namely, the Oseledec decomposition is holonomy invariant (see Proposition 6.1 (i)), whereas the other approach only tells us that the decomposition (48) is $g_{t}$-invariant.

We conclude the article with some remarks and open questions.

Remark 6.2. It seems interesting to relax the conditions imposed on Theorem 1.4, More concretely, we have the following three open questions.

Question 1. Is assertion (i) of Theorem 1.4 still true if the cocycle $\mathcal{A}$ is Hölder of order $\alpha \geq 2$ ?

Question 2. Is assertion (ii) of Theorem 1.4 still valid if the strong moderateness in Definition 2.8 is weakened as follows: a cocycle $\mathcal{A}$ is called strongly moderate if it is leafwise $\mathscr{C}^{2}$-differentiable cocycle and if there is a constant $c>0$ such that for every $(x, u) \in X \times \mathbb{P}^{d-1}(\mathbb{K})$, both $f_{x, u}$ and $\Delta f_{x, u}$ are moderate functions with constant $c$.

Question 3. Can one apply the result (or at least the approach) developed in this article to the holonomy cocycle of the whole regular part of a singular holomorphic foliation by hyperbolic Riemann surfaces? See [14, 15, 16, 22, 25, 27, 28] for a recent account on singular holomorphic foliations.

We hope to be able to come back some of these issues in forthcoming works.

\section{REFERENCES}

[1] Akcoglu, M. A.; Sucheston, L. A ratio ergodic theorem for superadditive processes. Z. Wahrsch. Verw. Gebiete 44 (1978), no. 4, 269-278.

[2] Ancona, A. Théorie du potentiel sur les graphes et les variétés. (French) [Potential theory on graphs and manifolds] École d'été de Probabilités de Saint-Flour XVIII-1988, 1-112, Lecture Notes in Math., 1427, Springer, Berlin, 1990.

[3] Berndtsson, Bo; Sibony, Nessim. The $\bar{\partial}$-equation on a positive current. Invent. Math. 147 (2002), no. 2, 371-428.

[4] Bonatti, Christian; Gómez-Mont, Xavier. Sur le comportement statistique des feuilles de certains feuilletages holomorphes. (French) [Statistical behavior of the leaves of certain holomorphic foliations] Essays on geometry and related topics, Vol. 1, 2, 15-41, Monogr. Enseign. Math., 38, Enseignement Math., Geneva, 2001.

[5] Bourbaki, N. Intégration, Vol. VI, Chapitre 6, Hermann, Paris, 1959.

[6] Candel, Alberto. Uniformization of surface laminations, Ann. Sci. École Norm. Sup. (4), 26 (1993), no. 4, 489-516.

[7] Candel, Alberto. The harmonic measures of Lucy Garnett, Adv. Math., 176 (2003), no. 2, 187-247.

[8] Candel, Alberto; Conlon, Lawrence. Foliations. II. Graduate Studies in Mathematics, 60. American Mathematical Society, Providence, RI, 2003. xiv +545 pp.

[9] Castaing, C.; Valadier, M. Convex analysis and measurable multifunctions. Lecture Notes in Mathematics, Vol. 580. Springer-Verlag, Berlin-New York, 1977. vii+278 pp. 
[10] Chavel, Isaac. Eigenvalues in Riemannian geometry. Including a chapter by Burton Randol. With an appendix by Jozef Dodziuk. Pure and Applied Mathematics, 115. Academic Press, Inc., Orlando, FL, 1984. xiv $+362 \mathrm{pp}$.

[11] Cranston, M. On the means of approach to the boundary of Brownian motion. Ann. Probab. 15 (1987), no. 3, 1009-1013.

[12] Deroin, Bertrand. Hypersurfaces Levi-plates immergées dans les surfaces complexes de courbure positive. (French) [Immersed Levi-flat hypersurfaces in complex surfaces of positive curvature] Ann. Sci. École Norm. Sup. (4) 38 (2005), no. 1, 57-75.

[13] Deroin, Bertrand; Dupont, Christophe. Topology and dynamics of laminations in surfaces of general type. J. Amer. Math. Soc. 29 (2016), no. 2, 495-535.

[14] Dinh, T.-C.; Nguyên, V.-A.; Sibony, N. Heat equation and ergodic theorems for Riemann surface laminations. Math. Ann. 354, (2012), no. 1, 331-376.

[15] Dinh, T.-C.; Nguyên, V.-A.; Sibony, N. Entropy for hyperbolic Riemann surface laminations I. Frontiers in Complex Dynamics: a volume in honor of John Milnor's 80th birthday, (A. Bonifant, M. Lyubich, S. Sutherland, editors), 569-592, (2014), Princeton University Press.

[16] Dinh, T.-C.; Nguyên, V.-A.; Sibony, N. Entropy for hyperbolic Riemann surface laminations II. Frontiers in Complex Dynamics: a volume in honor of John Milnor's 80th birthday, (A. Bonifant, M. Lyubich, S. Sutherland, editors), 593-622, (2014), Princeton University Press.

[17] Dunford, Nelson; Schwartz, Jacob T. Linear operators. Part I. General theory. With the assistance of William G. Bade and Robert G. Bartle. Reprint of the 1958 original. Wiley Classics Library. A WileyInterscience Publication. John Wiley \& Sons, Inc., New York, 1988. xiv+858 pp.

[18] Dynkin, E. B. Markov processes. Vols. I, II. Translated with the authorization and assistance of the author by J. Fabius, V. Greenberg, A. Maitra, G. Majone. Die Grundlehren der Mathematischen Wissenschaften, Bände 121, 122, Academic Press Inc., Publishers, New York; Springer-Verlag, BerlinGöttingen-Heidelberg, 1965 Vol. I: xii+365 pp.; Vol. II: viii+274 pp.

[19] Fornæss, J.-E.; Sibony, N. Riemann surface laminations with singularities. J. Geom. Anal., 18 (2008), no. $2,400-442$.

[20] Garnett, Lucy. Foliations, the ergodic theorem and Brownian motion. J. Funct. Anal. 51 (1983), no. 3, 285-311.

[21] Ghys, Étienne. Laminations par surfaces de Riemann. (French) [Laminations by Riemann surfaces] Dynamique et géométrie complexes (Lyon, 1997), ix, xi, 49-95, Panor. Synthèses, 8, Soc. Math. France, Paris, 1999.

[22] Glutsyuk, A.A. Hyperbolicity of the leaves of a generic one-dimensional holomorphic foliation on a nonsingular projective algebraic variety. (Russian) Tr. Mat. Inst. Steklova, 213 (1997), Differ. Uravn. s Veshchestv. i Kompleks. Vrem., 90-111; translation in Proc. Steklov Inst. Math. 1996, no. 2, 213, 83-103.

[23] Katok, Anatole; Hasselblatt, Boris. Introduction to the modern theory of dynamical systems. With a supplementary chapter by Katok and Leonardo Mendoza. Encyclopedia of Mathematics and its Applications, 54. Cambridge University Press, Cambridge, 1995. xviii+802 pp.

[24] Lyons Terry. A synthetic proof of Makarov's Law of the Iterated Logarithm. Bull. London Math. Soc. 22 (1990), no. 2, 159-162.

[25] Lins Neto A. Uniformization and the Poincaré metric on the leaves of a foliation by curves. Bol. Soc. Brasil. Mat. (N.S.), 31 (2000), no. 3, 351-366.

[26] Nguyên, Viêt-Anh. Oseledec multiplicative ergodic theorem for laminations. Mem. Amer. Math. Soc. 246 (2017), no. 1164, ix+174 pp.

[27] Nguyên, Viêt-Anh. Directed harmonic currents near hyperbolic singularities. Ergodic Theory Dyn. Syst. (2017), DOI: https://doi.org/10.1017/etds.2017.2, 18 pages.

[28] Nguyên, Viêt-Anh. Singular holomorphic foliations by curves I: Integrability of holonomy cocycle in dimension 2. math.DS, math.CV, math.DG, arXiv:1403.7688, 73 pages.

[29] Oseledec, V.-I. A multiplicative ergodic theorem. Lyapunov characteristic numbers for dynamical systems. Trans. Moscow Math. Soc., 19 (1968), 197-221.

[30] Sullivan, Dennis. Cycles for the dynamical study of foliated manifolds and complex manifolds. Invent. Math. 36 (1976), 225-255. 
[31] Walters, Peter. A dynamical proof of the multiplicative ergodic theorem. Trans. Amer. Math. Soc. 335 (1993), no. 1, 245-257.

Viêt-Anh Nguyên, Université de Lille 1, Laboratoire de mathématiques Paul Painlevé, CNRS U.M.R. 8524, 59655 Villeneuve d'Ascq Cedex, France.

Viet-Anh.Nguyen@math.univ-lille1.fr, http://www.math.univ-lille1.fr/ vnguyen 